\title{
Avulsions dentaires et kystectomies chez les patients présentant un déficit constitutionnel en facteurs de l'hémostase : conduite à tenir
}

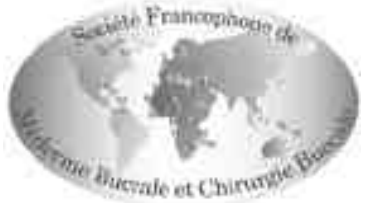 \\ SOCIÉTÉ FRANCOPHONE DE MÉDECINE BUCCALE \\ ET DE CHIRURGIE BUCCALE
}

\section{Groupe de travail}

Alp Alantar (Odontologiste, Paris), Coordonnateur Danielle Ginisty (Stomatologiste-Chirurgien

maxillo-facial, Paris)

Michel Hassin (Stomatologiste, Paris)

Antoine Lafuma (Méthodologiste, Paris)

Guy Princ (Stomatologiste-Chirurgien maxillofacial, Paris)

Natalie Stieltjes (Hématologue, Paris).

\section{Groupe de lecture}

Marie-Madeleine Baralle (Stomatologiste-

Chirurgien maxillo-facial, Lille)

J ean-Louis Blanc (Stomatologiste-Chirurgien maxillo-facial, Marseille)

Hervé Chambost (Hématologue, Marseille) Yves Commissionnat (Stomatologiste, Paris)

Christian Conseiller (Anesthésiste-Réanimateur, Paris)

Gilbert de Mello (Odontologiste, Rennes)

J oël Ferri (Stomatologiste-Chirurgien maxillo-facial, Lille)

\author{
Didier Gauzeran (Odontologiste, Paris) \\ Philippe Genet (Hématologue, Argenteuil) \\ Eric Gérard (Odontologiste, Metz) \\ Patrick Goudot (Stomatologiste-Chirurgien \\ maxillo-facial, Montpellier) \\ Vincent Laborie (Anesthésiste-Réanimateur, Paris) \\ Didier Luftman (Anesthésiste-Réanimateur, Paris) \\ Gabriel Malka (Stomatologiste-Chirurgien maxillo- \\ facial, Dijon) \\ Louis Maman (Odontologiste, Paris) \\ Daniel Perrin (Odontologiste, Dijon) \\ Laurence Pernice (Stomatologiste, La Garde) \\ Elisabeth Poidatz (Stomatologiste, Paris) \\ François Predine-Hug (Odontologiste, Brest) \\ J ean-P ierre Ragot (Stomatologiste, Paris) † \\ Yvon Roche (Odontologiste, Paris) \\ Marc Samama (Anesthésiste-Réanimateur, \\ Bobigny) \\ J acky Samson (Stomatologiste, Genève) \\ Christian Wang (Odontologiste, Nancy).
}

médecine buccale chirurgie buccale VOL. $11, \mathrm{~N}$ 2005 page 121

\section{Abréviations}

\author{
AP : Accord professionnel \\ CFH : Concentré de Facteurs de l'Hémostase \\ DCP : Déficit-Chirurgie-Patient \\ FH : Facteur de l'Hémostase \\ GRF : Gélatine-Résorcine-Formol
}

$\mathrm{NdP}$ : Niveau de preuve rFVIla : facteur VII recombinant activé SFM bCb : Société Francophone de Médecine Buccale et Chirurgie Buccale WFH : World Federation of Hemophilia

\section{Remerciements :}

Le groupe de travail tient à remercier pour leur collaboration à ce travail Mesdames Marie Claude LINCHET (secrétaire médicale, Hôpital Cochin), Geneviève J OLIVET (cadre infirmier, Hôpital Cochin) et Elisabeth TONNEL (manipulatrice en radiologie, Hôpital Cochin).

Demande de tirés à part :

Alp Alantar - Service de Stomatologie et Chirurgie maxillo-faciale de l'Hôpital Cochin - 27 rue du Faubourg Saint J acques - 75014 Paris - France Accepté pour publication le 14 janvier 2005 
médecine buccale chirurgie buccale

\section{RÉSUMÉ}

Contexte : L'existence de nombreux protocoles d'hémostase pour les avulsions dentaires chez les patients adultes ayant un déficit en facteur de l'hémostase $(\mathrm{FH})$ a conduit le Service de Stomatologie et de Chirurgie Maxillo-faciale et le Centre de Traitement des Hémophiles du Groupe hospitalier Cochin-Saint-Vincent-de-Paul-La-Roche-Guyon (Paris) à proposer des recommandations pour leur prise en charge.

Objectif : Etablir des recommandations pour les avulsions dentaires et les kystectomies chez les patients atteints d'un déficit congénital (mineur, modéré ou sévère) en $\mathrm{FH}$ revient à définir le protocole d'hémostase visant à réduire le recours aux concentrés de facteurs de l'hémostase (CFH), tout en assurant la sécurité et le confort pour le patient.

Méthodologie : La méthodologie d'élaboration des recommandations repose sur une revue et une analyse critique des données de la littérature scientifique par un Groupe de travail permettant de définir, sur la base du niveau de preuves scientifiques (NdP 1-2-3-4) et du jugement argumenté du groupe de travail, des recommandations (Grades A-B-C). Les recommandations ainsi élaborées sont validées par un groupe de lecture indépendant.

Matériel : Les données sources pour l'élaboration des recommandations sont issues :

1) d'une recherche automatisée sur la banque de données Medline couvrant la période 1966-2003;

2) d'une recherche manuelle dans les quintessences des 23 et $24^{\text {e }}$ congrès de la World Federation of Hemophilia (WFH) ;

3) la lecture de six ouvrages français et anglais de pathologie et de chirurgie buccales.

Résultats : La lecture de 279 articles, de 13 résumés de congrès et de 6 ouvrages ont permis d'établir les recommandations générales suivantes :

1) app roche pluridisciplinaire associant un hématologue et un stomatologiste ou un odontologiste : 2) protocole d'hémostase adapté au type et à la sévérité du déficit en $\mathrm{FH}$, ou au type de chirurgie - dent(s) sur l'arcade, dent(s) incluse(s), avulsions multiples... - tout en tenant compte de la compliance du patient (critères DCP).

Les recommandations sont présentées selon la chronologie des différents phases opératoires.
En pré-opératoire :

1) prescription d'acide tranexamique et d'antibiotiques ;

2.a) pour les patients ayant un déficit sévère avec ou sans inhibiteur ou pour des avulsions sous anesthésie générale : perfusion quasi systématique du FH manquant dans I'heure précédant I'intervention : facteur VIII pour I'hémo philie A, facteur IX pour I'hémophilie $B$, facteur Willebrand pour la maladie de Willebrand de type 3 ;

2.b) pour les patients ayant un déficit modéré ou mineur : administration de desmopressine (même si la réponse est partielle) pour l'hémophilie $A$ et la maladie de Willebrand, sans administration systématique de $\mathrm{FH}$.

En per-opératoire:

1) privilégier l'anesthésie locale faite avec un anesthésique à liaison amide associé à un vasoconstricteur ;

2) avulsions multiples réalisées en plusieurs séances, en général par hémi-arcade ;

3) avulsion ou kystectomie la moins délabrante possible, complétée par un parage minutieux de la plaie ;

4) lambeaux strictement muco-périostés ;

5) pour réaliser l'hémostase locale on associe suture et/ou colle chirurgicale avec un matériau tels que des mèches d'oxycellulose ;

6) pour comp léter l'hémostase locale, mise en place de gouttières hémostatiques ;

7) anesthésie générale pour les actes de chirurgie invasive (dents incluses en position ectopique, avulsions multiples touchant les différents quadrants, édentation totale bimaxillaire, kystectomie) sous réserve d'une correction préalable du déficit en $\mathrm{FH}$ par un traitement substitutif.

En post-opératoire : 1 ) perfusion(s) de $\mathrm{CFH}$ et/ou de desmopressine à la demande en cas de saignement ou à l'appréciation du chirurgien et de I'hématologue ;

2) prescription d'un antalgique et d'acide tranexamique ;

3) remise au patient d'une fiche de conseils postopératoires comportant un numéro de téléphone répondant $24 \mathrm{~h} / 24$ aux appels d'urgence ;

4) consultation à 7. 


\section{SUMMARY}

Context: A variety of protocols has been proposed for the management of adult patients with hemostasis factor (HF) deficiency undergoing dental avulsions. Therefore the Haemophilia Centre and the Oral and Maxillofacial Surgery Unit of Hospital Cochin elaborated Health Care Recommendations $(H C R)$ for the management of these patients.

Objective: The aim of these HCR is to propose clinical practice guidelines in order to reduce hemostasis factor concentrate (HFC) requirement in such patients undergoing dental avulsions and concurrently to improve their safety and comfort. Method: The methodology was based on literature review and critical appraisal by a multidisciplinary working group. Once the guidelines had been defined, the document was submitted to a reviewer group for peer review and agreement. Data have been collected by:

1) a literature search using Medline (from 1966 up to 2003);

2) the analysis of the 23rd and 24th World Federation of Hemophilia congress;

3) the lecture of six French and English oral pathology and oral surgery books.

Results: Data have been obtained from 279 articles, 13 abstracts of congress and 6 textbooks; the HCR are the following :

1) multid isciplinary collaboration between the haematologist and the oral surgeon;

2) pragmatic approach: the choice of the hemostatic technique depends on the severity of the $\mathrm{HF}$ deficiency, the type of surgery and the patient's compliance (DSP criterias).

\section{Preoperatively:}

1) tranexamic acid and antibiotics prescription; 2.a) severe deficiency with or without inhibitors or avulsions under general anaesthesia : perfusion of HFC one hour before surgery;

2.b) moderate and minor deficiency: eventually single preoperative infusion of $\mathrm{HF}$ for haemophilia $B$ and preoperative desmopressin in patients with haemophilia $\mathrm{A}$ and Willebrand disease.

Peroperatively:

1) local anaesthesia with amid solution plus vasoconstrictor;

2) multiple avulsions performed on different days; 3) surgical procedure as less traumatic as possible;

4) periosteal flap;

5) association of sutures and/or surgical glue to a local hemostatic as: oxycellulose;

6) when possible, use of a splint;

7) in case of hard surgery (complete maxillary and mandibulary edentation, wisdom teeth avulsion, cystectomy) and according to the compliance of the patient a general anesthesia with provided correction of hemostasis can be indicated.

Postoperatively:

1) (re)infusion of HFC and/or desmopressin in case of secondary bleeding uncontrollable by local means or according to oral surgeon or haematologist;

2) tranexamic acid and antalgic drugs on demand; 3) postoperative written advice including a phone number in case of emergency;

4) follow-up on D7. médecin€ buccale chirurgie buccale VoL. $11, \mathrm{~N}$ 2005 page 123 
médecine buccale chirurgie buccale
Chez les patients atteints d'un déficit constitutionnel en facteur(s) de l'hémostase $(\mathrm{FH})$, les avulsions dentaires et les kystectomies représentent un acte comportant un haut risque hémorragique. Divers protocoles ont été proposés pour réaliser ces interventions : utilisation d'hémostatiques locaux (mèches d'oxycellulose, éponges hémostatiques, sutures, colles chirurgicales), de gouttières hémostatiques, d'antifibrinolytiques locaux et de traitements administrés par voie générale (concentrés de facteurs de l'hémostase - CFH -, desmopressine, antifibrinolytiques).

L'objectif de cette mise au point, fondée sur l'analyse des différents protocoles publiés pour la réalisation de ces interventions, est d'élaborer des recommandations pour les patients présentant un déficit constitutionnel en $\mathrm{FH}$. Le cas particulier des enfants (conditions locorégionales spécifiques, recours fréquent à l'anesthésie générale) n'est pas abordé dans cette étude.

\section{MÉTHODOLOGIE GÉNÉRALE}

La méthodologie d'élaboration des recommandations repose sur la revue et l'analyse critique, par le groupe de travail plurid isciplinaire, des données de la littérature scientifique permettant, selon le niveau de preuve scientifique (NdP 1-2-3-4) d'établir des recommandations (Grades A-B-C) (Guyatt et coll., 1995 ; ANAES, 1999) (Tab. 1).
Compte tenu du faible niveau de preuve (études de cas, séries rétrospectives...), les résumés des communications internationales des 23 et $24^{\mathrm{e}}$ congrès de la World Federation of Hemophilia (WFH) publiés dans la revue Haemophilia (The Official J ournal of the World Federation of Hemophilia) et le dépouillement de six ouvrages de pathologie et chirurgie buccales de langues française et anglaise ont également été pris en compte. Le texte a été relu par les membres d'un groupe de lecture pluridisc iplinaire indépendant, composé de praticiens hospitaliers. L'existence éventuelle d'un désaccord majeur et argumenté d'un ou de plusieurs membres des deux groupes sur une ou plusieurs recommandations principales doit être mentionnée dans le texte final. En raison de données scientifiques partielles ou absentes ne permettant pas de répondre à l'ensemble des questions, le principe de la méthode Delphi (in J eremy et J ones, 1995) a été appliquée : renvoi du texte au groupe de lecture afin d'obtenir un accord professionnel (AP) sur les points concernés. Après intégration des différentes remarques et relecture par le groupe de travail, les recommandations ont été présentées aux $47^{\mathrm{e}}$ J ournées de la Société Francophone de Médecine Buccale et Chirurgie Buccale (SFMbCb), le 22 mai 2004 à M ontpellier. Le texte a été soumis à nouveau au groupe de lecture pour la validation finale.

Tableau 1 : Grade des recommandations (ANAES, 2000)

Niveau de preuve scientifique fourni par la littérature

Niveau 1:

- Essais comparatifs randomisés de forte puissance

- Méta-analyse d'essais comparatifs randomisés

- Analyse de décision basée sur des études bien menées

Niveau 2 :

- Essais comparatifs randomisés de faible puissance

- Etudes comparatives non randomisées bien menées

- Etudes de cohorte

Niveau 3 :

- Etudes cas-témoin

Niveau 4 :

- Etudes comparatives comportant des biais importants

- Etudes rétrospectives

- Séries de cas

- Etudes épidémiologiques descriptives (transversales, longitudinales)
Grade des recommandations

A

Preuve scientifique établie

B

Présomption scientifique

C

Faible niveau de preuve scientifique 


\section{Stratégie de la recherche documentaire}

La recherche automatisée a été effectuée sur la banque de données MEDLINE (PubMed) couvrant la période 1966-2003, sans restriction de langue. Les mots du MESH suivants ont été interrogés :

- bleeding

- oral hemorrhage

- tooth avulsion

- oral surgery

Les mots clés initiaux ont été croisés avec :

- splint

- prevention

- inhibitor

- tranexamic acid

- aprotinin

- desmopressin

- blood coagulation factor deficiency

- hemophilia

- willebrand

Avec ces interrogations (toutes recherches confondues avec possibilité de redondance), 279 références ont été obtenues. Les références citées correspondent aux articles exploitables en langues anglaise et française. La recherche manuelle a été basée sur le dépouillement des quintessences des 23 et $24^{\text {e }}$ congrès de la WFH a permis de retrouver 13 résumés abordant ce sujet. Les données recueillies dans les six ouvrages ont été aussi prises en compte.

\section{Questions retenues}

1. Quelle(s) techniques (s) d'anesthésie recommander?

2. Quelle(s) technique(s) d'avulsion et de kystectomie utiliser?

3. Quelles techniques hémostatiques locales mettre en œuvre?

4. Quelles sont les indications d'un traitement par voie générale?

5. Comment envisager la surveillance et les traitements post-opératoires?

\section{AVULSIONS DENTAIRES ET KYS- TECTOMIES CHEZ LES PATIENTS PRESENTANT UN DEFICIT CONSTI- TUTIONNEL EN FACTEUR(S) DE L'HEMOSTASE (FH) : CONDUITE A TENIR (Tab. 2)}

Avant tout :

- Il doit y avoir une collaboration entre l'odontologiste ou le stomatologiste et l'hématologue pour le choix du protocole qui est fait sur la base du bilan hématologique (caractérisation du déficit : type et sévérité du déficit en $\mathrm{FH}$, présence ou non d'un inhibiteur), de l'examen clinique (ouverture buccale, importance du délabrement dentaire, état des tissus mous, degré d'édentation, taille du kyste) et radiographique (orthopantomogramme). Le protocole adopté est expliqué au patient [AP];

- Il faut évaluer les facteurs de risque (critères DCP, Tab. 3) qui sont déterminants pour le protocole :

- Déficit (D) : par exemple, un patient hémophile sévère et un patient atteint d'une maladie de Willebrand modérée ne peuvent s'inscrire dans le même protocole ;

- Chirurgie (C) : avulsions uniques ou multiples de dents sur l'arcade ou de dents incluses, taille du kyste, présence d'une réaction inflammatoire ;

- Compliance du patient (P) : évaluation de son observance du traitement, de son aptitude à l'auto- traitement, de sa capacité à comprendre la procédure, de son éloignement du centre hospitalier, de ses habitudes socio-professionnelles [Grade C].

\section{Recommandations pré-opératoires}

\subsection{Indication d'une hospitalisation}

Elle dépend du type et de la sévérité du déficit en $\mathrm{FH}$, du type d'intervention chirurgicale, du profil psychosocial et de la compliance du patient, et de l'éloignement du domicile du patient [AP].

\subsection{Prescriptions}

Elles sont adaptées à chaque cas :

- prescription d'antibiotiques en fonction de l'état général du patient $(\mathrm{VIH}$, diabète non équilibré... ) médecin€ buccale chirurgie buccale VOL. $11, \mathrm{~N}$ 2005 page 125 
et de l'état infectieux du site opératoire. Elle est systématique pour une alvéolectomie, une kystectomie et lors de la mise en place de gouttières hémostatiques [Grade $\mathbf{C}$ ] ;

- arrêt de tout traitement anti-inflammatoire non stéroïdien au moins 48 heures avant l'intervention [Grade C] ; on prescrit l'antibioprophylaxie habituellement conseillée en odonto-stomatologie :

- pénicilline $A$ + inhibiteur des bêtalactamases, 2 g.j. . $^{-1}$ per os pendant $5 \mathrm{j}$;

- en cas d'allergie à la pénicilline : clindamycine + gentamicine, $600 \mathrm{mg}$ en pré-opératoire, $600 \mathrm{mg} .6 \mathrm{~h}^{-1}$ pendant $48 \mathrm{~h}$ puis per os pendant $5 \mathrm{j}$.

- prescription d'acide tranexamique en rinçage ( 3 min. $\left.6 \mathrm{~h}^{-1}\right)$ et per os ( 2 à 4 g..$\left.^{-1}\right)$ pendant $7 \mathrm{j}$ à partir du jour de l'intervention [Grade $\mathbf{C}$ ].

médecine buccale chirurgie buccale

\subsection{Correction de l'hémostase}

Elle dépend du déficit en $\mathrm{FH}$.

Pour un déficit modéré ( $1 \% \leq F H<7 \%$ ) ou mineur (FH $\geq 7 \%)$

On prescrit de la desmopressine lorsque le test au Minirin ${ }^{\circledR}$ est positif. L'administration se fait selon deux modalités :

- soit M inirin ${ }^{\circledR}: 0,3 \mu \mathrm{g} \cdot \mathrm{kg}^{-1}$ dans $50-100 \mathrm{ml}$ de sérum physiologique en perfusion IV lente en 15 à 30 min pour l'hémophilie $A$ et la maladie de Willebrand (sauf pour le type 3 ), avec surveillance du pouls et de la tension artérielle, et restriction des apports hydriques [Grade $\mathbf{C}$ ]. Le M inirin ${ }^{\circledR}$ doit être utilisé avec prudence chez les patients présentant une affection cardiovasculaire. Lors d'administrations répétées, il faut effectuer une surveillance étroite du pouls, de la tension artérielle, de la diurèse, de la natrémie et de la restriction hydrique [Grade $\mathbf{C}$ ].

- soit Octim ${ }^{\circledR}$ spray nasal : 1 pulvérisation unique de $150 \mu \mathrm{g}$ pour les patients de moins de $50 \mathrm{~kg}$ et 2 pulvérisations pour les patients de plus de $50 \mathrm{~kg}$ [Grade $\mathbf{C}$ ] avec une surveillance identique à celle réalisée pour le Minirin ${ }^{\circledR}$.

Pour un déficit sévère $(\mathrm{FH}<1 \%)$ sans inhibiteur Une dose unique de CFH est injectée dans l'heure précédant l'intervention :

- pour un déficit en facteur VIII : 20-50 UI. $\mathrm{kg}^{-1}$ de facteur VIII [Grade C] en 5-10 min [AP];
- pour un déficit en facteur IX : 30-60 UI. $\mathrm{kg}^{-1} \mathrm{de}$ facteur IX [Grade C] en 5-10 min [AP].

Pour une hémophilie avec inhibiteur

- $90 \mu \mathrm{g} \cdot \mathrm{kg}^{-1}$ de rFVlla (NovoS even ${ }^{\circledR}$ ) (Grade C] en 5-10 min, juste avant le geste opératoire [AP] ou, - 60-100 U. $\mathrm{kg}^{-1}$ de Feib ${ }^{\circledR}$ en 30-45 min, juste avant le geste opératoire [AP].

\subsection{Patients opérés sous anesthésie générale}

Les mesures sont les suivantes:

- indications de l'anesthésie générale : elle est conseillée pour les interventions à haut risque hémorragique, avulsion des dents incluses ectopiques, avulsions multiples, kystectomie, patients pusillanimes [Grade C];

- prescriptions pré-opératoires : ce sont les mêmes que pour les interventions sous AG [Grade $\mathbf{C}$ ]; - correction pré-opératoire du trouble de la coagulation avec maintien des $\mathrm{FH}$ pendant quelques heures ou plus pour prévenir le risque de saignement nasal ou endo-trachéal s'il y a une intubation naso-trachéale ou oro-trachéale [AP] ;

- pour la chirurgie et l'hémostase locale : on ap plique les mesures préconisées pour l'anesthésie locale [Grade $\mathbf{C}$ ].

\section{Recommandations per-opératoires}

\subsection{Indication de l'anesthésie locale ou de l'anesthésie générale}

L'anesthésie locale est réalisé avec un anesthésique du groupe amide à $2-3 \%$, contenant un vasoconstricteur (1/100.000 à 1/200.000), en utilisant des aiguilles fines $(0,3$ ou $0,4 \mathrm{~mm}$ de diamètre) et en réalisant une aspiration de contrôle avant l'injection [AP].

L'anesthésie générale se fait, si possible, au masque laryngé [Grade $\mathbf{C}$ ]

\subsection{Réalisation de l'intervention}

- pour les avulsions multiples, il faut procéder de façon sectorielle par hémi-arcade ; le délai séparant deux séances est laissé à l'appréciation de I'opérateur qui prend en compte l'évaluation de la difficulté des avulsions, les suites opératoires de l'intervention précédente et les doléances du patient [Grade B] ; 
- avulsion la moins délabrante possible : les mouvements de circumduction, de rotation ou d'oscillation doivent être d'amplitude limitée ; la séparation des racines préférée à l'alvéolectomie [AP] ;

- pour les interventions avec lambeaux : il faut toujours faire un lambeau strictement muco-périosté [AP] ;

- toujours effectuer un parage minutieux de la plaie [AP].

\subsection{Protocole d'hémostase locale}

Les mesures sont les suivantes:

- sutures résorbables et colle sont associés à un matériau d'hémostase tel que des mèches d'oxycllulose [AP] ;

- compression avec des compresses, stériles imbibées d'acide tranexamique, pendant 5 à 20 min [Grade C];

- contrôle de l'hémostase (absence d'hémorragie dans les 5 min qui suivent la dépose des compresses) [AP];

- mise en place de gouttières hémostatiques qui doivent être stables, légèrement compressives, atraumatiques et ne pas gêner la fonction (phonation et alimentation) [AP]. Si cette mise en place s'avère impossible (dent de sagesse incluse, édentation subtotale, dents restantes non rétentives, refus du patient) I'utilisation d'une colle associée à un matériau hémostatique résorbable et des sutures est indiquée [Grade C ].

\section{Recommandations post-opératoires}

\subsection{Protocole pour les perfusions de CFH} Leur indication est adaptée à chaque cas :

- les perfusion(s) post-opératoire(s) de CFH sont faites à la demande lors d'un saignement postopératoire ou laissée(s) à l'appréciation du chirurgien et de l'hématologue (type et sévérité du déficit, type de chirurgie, compliance du patient), voire du patient lui-même pour les patients hémophiles sévères pratiquant l'auto-traitement [Grade $\mathbf{C}$ ] ; - pour les hémophiles avec inhibiteur : $90 \mu \mathrm{g} . \mathrm{kg}^{-1}$ de rFVIla (NovoSeven ${ }^{\circledR}$ ) en 5-10 min, 2 h et éven- tuellement $4 \mathrm{~h}$ après la première perfusion ou 60 100 U. $\mathrm{kg}^{-1}$ de Feiba ${ }^{\circledR}$ en 30-45 min [Grade C].

\subsection{Prescriptions}

Elles comportent :

- des antalgiques : paracétamol ou dextropropoxyphène [Grade $\mathbf{C}$ ] ;

- l'acide tranexamique en rinçage ( $\left.3 \mathrm{~min} .6 \mathrm{~h}^{-1}\right)$ et per os (2 à $4 \mathrm{~g}^{j^{-1}}$ ) pendant $7 \mathrm{j}$ [Grade C ] ;

- une antibiothérapie (pénicilline/acide clavulanique ou macrolides/métronidazole; $2 \mathrm{~g} . \mathrm{j}^{-1}$ ) pendant 8 j ou plus selon la durée de port des gouttières hémostatiques pour prévenir le risque infectieux ou lorsqu'il existe une infection du site opératoire [Grade C].

\subsection{Notice de conseils}

Les conseils sont donnés verbalement et par écrit : - l'alimentation doit être liquide ou semi-liquide, et tiède ou froide pendant 2 à 8 jours [Grade $\mathbf{C}$ ] ; - arrêt du tabac pendant la phase de cicatrisation [Grade C ] ;

- pas de bains de bouche intempestifs [GradeC]; - en cas de saignement : il faut mordre durant 20 min sur une compresse imprégnée d'une ampoule (10 ml) d'acide tranexamique et renouveler la procédure si nécessaire [Grade $\mathbf{C}$ ] ;

- communiquer un numéro de téléphone répondant 24h/24 aux appels d'urgence.

\subsection{Patients porteurs de gouttières hémo- statiques}

- lors d'un saignement, le patient doit mordre sur la gouttière pendant 20 min [AP] ;

- si la gouttière est mobile, blesse ou lors d'un saignement prolongé malgré la gouttière, le patient doit revenir en consultation pour une reprise de l'hémostase locale [Grade $\mathbf{C}$ ] : dépose et éventuellement nettoyage, puis rescellement de la gouttière avec un ciment chirurgical ou repose d'une nouvelle gouttière réalisée extemporanément [AP] ;

- le patient doit être revu une semaine après l'intervention pour contrôler la cicatrisation et l'hémostase, et déposer les gouttières [Grade $\mathbf{C}$ ]. médecin€ buccale chirurgie buccale VOL. $11, \mathrm{~N}$ 2005 page 127 
Tableau 2 : Conduite à tenir lors des avulsions dentaires et des kystectomies chez les patients présentant un déficit constitutionnel en facteur de l'hémostase (FH)

\begin{tabular}{|c|c|c|}
\hline PRE-OPERATOIRES & PER-OPERATOIRES & POST-OPERATOIRES \\
\hline \multicolumn{3}{|c|}{ Recommandations générales } \\
\hline $\begin{array}{l}\text { Prescription } \\
\text { - antalgiques } \\
\text { - antibiotiques } \\
\text { - acide tranexamique } \\
\text { - AINS contre-indiqués } \\
\text { - éventuellement corticothérapie brève }\end{array}$ & $\begin{array}{l}\text { Anesthésie locale } \\
\text { Amide } 2-3 \% \text {, vasoconstricteur } \\
(1 / 100000 \text { à } 1 / 200000) \\
\text { Aiguilles fines }(0,3-0,4 \mathrm{~mm}) \\
\text { Aspiration de contrôle } \\
\text { Avulsion } \\
\text { Multiples par hémi-arcades } \\
\text { Le moins délabrant possible } \\
\text { Séparation radiculaire préférable } \\
\text { à l'alvéolectomie } \\
\text { Lambeaux } \\
\text { D'emblée muco-périostés } \\
\text { Parage } \\
\text { Minutieux } \\
\text { Hémostase locale } \\
\text { Association suture-colle, } \\
\text { à un matériau d'hémostase } \\
\text { tel que des mèches d'oxycellulose }\end{array}$ & $\begin{array}{l}\text { Immédiates } \\
\text { Compression avec } \\
\text { Exacyl }{ }^{\circledR} \text { pendant } 5-20 \text { min } \\
\text { Contrôle de l'hémostase } \\
5 \text { min post-opératoires } \\
\text { Ultérieures } \\
\text { Poursuite des antibiotiques } \\
\text { et de l'acide tranexamique } \\
\text { (Ré) administration de FH } \\
\text { ou de desmopressine si nécessaire } \\
\text { Contrôle à J } 7 \\
\text { Notice de conseils } \\
\text { Alimentation liquide ou } \\
\text { semi-liquide } \\
\text { boissons froides } \\
n^{\circ} \text { de téléphone }\end{array}$ \\
\hline \multicolumn{3}{|c|}{ Recommandations spécifiques pour les déficits modérés ou mineurs } \\
\hline $\begin{array}{l}\text { En cas d'hémophilie modérée ou minec } \\
\text { Willebrand (sauf type } 3 \text { ) chez le sujet ré } \\
\text { desmopressine : Minirin }{ }^{\circledR} 0,3 \mu \mathrm{g} \cdot \mathrm{kg}^{-1} / 5 \\
\text { Perfusion IV lente en } 15-30 \mathrm{~min} \text { ou spra } \\
1 \text { pulvérisation unique de } 150 \mu \mathrm{g} \text { pour } \\
\text { et } 2 \text { pulvérisations pour ceux }>50 \mathrm{~kg} \text { ) } \\
\text { Surveillance du pouls, de la TA et restric } \\
\text { Surveillance plus rapprochée en cas d'a } \\
\text { Administration de CFH en fonction des }\end{array}$ & $\begin{array}{l}\text { ou de maladie de } \\
\text { ndeur à la } \\
100 \mathrm{ml} \text { de sérum physiologique } \\
\text { lasal }\left(0 \mathrm{ctim}{ }^{\circledR}\right. \\
\text { patients }<50 \mathrm{~kg} \\
\text { n des apports hydriques } \\
\text { ninistrations répétées } \\
\text { tères DCP }\end{array}$ & $\begin{array}{l}\text { Perfusions de CFH } \\
\text { ou (ré)administration de } \\
\text { Minirin }{ }^{\circledR} \text { en fonction des } \\
\text { critères DCP et de } \\
\text { l'évolution clinique }\end{array}$ \\
\hline \multicolumn{3}{|c|}{ Recommandations spécifiques pour les déficits sévères } \\
\hline $\begin{array}{l}\text { Une dose de CFH injectée dans I'heure } \\
\text { Hémophilie A } \\
\text { 20-50 UI. kg }{ }^{-1} \text { de facteurs VIII en 5-10 m } \\
\text { Hémophilie B } \\
\text { 30-60 UI. } \mathrm{kg}^{-1} \text { de facteurs IX en 5-10 mir }\end{array}$ & écédant l'intervention & $\begin{array}{l}\text { Perfusion de CFH } \\
\text { fonction des critères DCP } \\
\text { et de l'évolution clinique. }\end{array}$ \\
\hline \multicolumn{3}{|c|}{ Recommandations spécifiques pour l'hémophilie avec inhibiteur } \\
\hline $\begin{array}{l}\text { Perfusion } \\
\text { Pour les patients ayant reçu en pré-opé } \\
90 \mu \mathrm{g} \cdot \mathrm{kg}^{-1} \text { de rFVIla en } 5-10 \text { min }\end{array}$ & oire du NovoSeven ${ }^{\circledR}$ & $\begin{array}{l}\text { Perfusion } \\
\text { Réadministration de rFVIla ou Feiba } \\
\text { en fonction des critères DCP et } \\
\text { de l'évolution clinique }\end{array}$ \\
\hline \multicolumn{3}{|c|}{ Avulsions sous AG } \\
\hline $\begin{array}{l}\text { Correction pré-opératoire des trouk } \\
\text { nécessaire (risque hémorragique lié } \\
\text { Prescription d'antibiotiques } \\
2 \mathrm{~g} \text { en pré-opératoire puis } 1 \mathrm{~g}, 6 \mathrm{~h} \text { postc } \\
\text { si allergie à la pénicilline : clindamycine/ } \\
600 \mathrm{mg} \text { puis } 600 \mathrm{mg} 6 \mathrm{~h} \text { post op,relais } \\
\text { Technique d'anesthésie : préférence }\end{array}$ & $\begin{array}{l}\text { s de l'hémostase } \\
\text { l'intubation) } \\
\text { ratoire, relais per os } 8 \mathrm{j} \text {; } \\
\text { ntamycine, } \\
\text { ros pendant } 8 \text { j. } \\
\text { ur le masque laryngé }\end{array}$ & $\begin{array}{l}\text { Perfusions post-opératoires } \\
\text { Perfusions post-opératoires en cas } \\
\text { de traumatisme à l'intubation }\end{array}$ \\
\hline
\end{tabular}


Tableau 3 : Critères DCP [Grade C ]

\section{Déficit (D)}

- type et sévérité du déficit

- présence d'anticorps circulants

\section{Chirurgie (C)}

- avulsions uniques ou multiples

- avulsion de dents sur l'arcade ou de dents incluses

- kystectomie

- présence d'un état inflammatoire

\section{Patient (P)}

- observance du traitement

- aptitude à l'auto-traitement

- capacité à comprendre la procédure

- éloignement d'un centre hospitalier

- habitudes socio-professionnelles 
médecine buccale chirurgie buccale

\section{ARGUMENTAIRE}

\section{Epidémiologie et pathogénie des défi- cits en facteurs de l'hémostase (FH)}

(Goudemand, 1997 ; Sonis et Musselman, 1982 ; Heim et coll., 2002 ; Aledort, 1994 ; Aledort et coll., 1994 ; Rodeghiero et coll., 1987 ; Sadler et coll., 2000 ; Mannucci et coll., 1981 ; Santagostino et Mannucci, 2000 ; Stubbs et Lloyd, 2001 ; Federici et coll., 2002 ; B rewer et coll., 2003).

Les défic its constitutionnels en $\mathrm{FH}$ regroupent plusieurs pathologies au premier rang desquelles se trouvent l'hémophilie et la maladie de Willebrand. L'hémophilie résulte d'un défaut de synthèse du facteur VIII (hémophilie A) ou du facteur IX (hémophilie $B$ ) de la coagulation. Les gènes codant pources facteurs sont situés sur le chromosome $X$, ce qui explique que cette maladie, à transmission récessive, touche uniquement les sujets mâles. II s'agit d'une maladie rare (un nouveau-né mâle atteint sur 5000). Le diagnostic biologique repose sur la mise en évidence d'un allongement isolé du TCA (temps de céphaline activé) avec un taux abaissé, voire indétectable, de facteur VIII ou de facteur IX. Selon I'importance du déficit, on distingue les formes sévères (taux de facteur VIII ou de facteur IX $<1 \%$ ), les formes modérées ( $1 \% \leq$ taux de facteur VIII ou de facteur IX $<7 \%$ ) et les formes mineures (taux de facteur VIII ou de facteur IX $\geq 7 \%$ ). L'expression clinique est en corrélation directe avec l'intensité du déficit. Dans l'hémophilie sévère, les premiers accidents hémorragiques surviennent très tôt dans l'enfance, spontanément ou après des traumatismes mineurs. Les manifestations cliniques les plus typiques sont les hémarthroses, qui touchent les chevilles, les genoux et les coudes. Leur répétition aboutit assez rapidement à la constitution d'une arthropathie hémophilique qui conditionne le pronostic fonctionnel. Les hématomes musculaires sont généralement post-traumatiques. On peut également observer toutes les autres manifestations secondaires à un trouble de l'hémostase : ecchymose post-traumatique, saignement prolongé après blessure, avulsion dentaire ou intervention chirurgicale, hémorragie cérébrale... Dans I'hémophilie modérée ou mineure, les manifestations surviennent généralement après un traumatisme ou lors d'une intervention chirurgicale. Un saignement prolongé après avulsion dentaire ou plaie de la muqueuse buccale est fréquemment retrouvé dans les antécédents de sujets atteints d'hémophilie mineure ; il constitue souvent l'incident qui amène à faire le diagnostic de la maladie.

Le pronostic et la qualité de vie des patients hémophiles, surtout ceux atteints de la forme sévère, ont été transformés par la possibilité d'un traitement substitutif à partir des années 60-70. L'apport du FH déficitaire par transfusion de sang total, puis de plasma frais congelé et maintenant de concentrés de facteur VIII ou de facteur IX ayant un haut degré de pureté, permet le traitement et la prévention des accidents hémorragiques et vise de plus en plus à prévenir le développement de l'arthropathie hémophilique. Ces traitements ont malheureusement été responsables de multiples contaminations virales (hépatite $B$, hépatite $C, V I H$ ) jusqu'au milieu des années 80. Depuis 1987, la mise au point de méthodes d'inactivation virale pour les dérivés $d u$ sang et la production de $\mathrm{FH}$ d'origine recombinante ont permis de prévenir toute contamination par ces virus. Néanmoins, le risque résiduel de transmission d'éventuels agents infectieux émergeants ne peut pas être totalement exclu : il justifie le maintien d'une veille sanitaire attentive.

Les patients atteints d'hémophilie A mineure peuvent être traités par desmopressine. Son administration permet en général une élévation du taux de facteur VIII (multiplié par 3 à 4 une demi-heure après l'administration intraveineuse de desmopressine), persistant pendant quelques heures (retour du taux de facteur VIII à la valeur initiale en 4 à 8 heures).

Certains patients hémophiles développent des inhibiteurs contre le facteur défic itaire après un nombre variable de transfusions de FH. Ces inhibiteurs ou anticorps anti-facteur VIII ou anti-facteur IX apparaissent chez 20 à $30 \%$ des hémophiles $A$ sévères, plus rarement chez les hémophiles $A$ modérés ou mineurs, et chez 1 à $3 \%$ des hémophiles $B$ sévères. Ils constituent actuellement la principale complication iatrogène de l'hémophilie. Le traitement substitutif devient 
alors inefficace : pour prévenir ou traiter les complications hémorragiques, il est nécessaire de recourir à l'administration de fractions coagulantes activées dont l'efficacité thérapeutique est moins satisfaisante et le maniement plus délicat. Des traitements assez lourds pour induire une tolérance immune visant à faire disparaître les inhibiteurs peuvent être tentés mais leur succès est inconstant et souvent long à obtenir. Les hémophiles avec inhibiteurs constituent donc une population à haut risque hémorragique lors de traumatismes ou d'actes chirurgicaux dans la mesure où le contrôle de l'hémostase est plus aléatoire.

La maladie de Willebrand est la plus fréquente des maladies hémorragiques constitutionnelles (incidence 1/1000). Elle est caractérisée par un déficit quantitatif ou qualitatif en facteur Willebrand. Elle se transmet sur le mode autosomal et s'exprime selon un mode dominant ou récessif selon le type. La majorité des maladies de Willebrand, dues à un déficit quantitatif modéré (maladie de Willebrand type 1), sont peu symptomatiques : il existe une tendance hémorrag ique variable, intéressant principalement les muqueuses (ménorragies, épistaxis, saignements après avulsions dentaires), le plus souvent en présence de facteurs locaux favorisants. Les déficits qualitatifs (maladie de Willebrand type 2) ont une expression clinique parfois plus importante. Les variants de type $2 \mathrm{~N}$, résultant d'un défaut de liaison du facteur Willebrand au facteur VIII, se présentent comme des hémophilies mineures (abaissement isolé du taux de facteur VIII). Les formes sévères (type 3 ), récessives, très rares (1 cas pour 1 million de naissances), sont caractérisées par un taux de facteur Willebrand indétectable et un taux de facteur VIII très bas $(<5 \%)$. La symptomatologie associe des saignements muqueux fréquents à des manifestations de type hémophilique.

Dans la majorité des cas de maladie de Willebrand, l'administration de desmopressine permet une élévation transitoire des taux du facteur Willebrand et du facteur VIII (qui sont multipliés par 3 ou 4), assurant le contrôle de la plupart des saignements. Néanmoins, dans le type 3 , dans certains types 2 , et chez les patients ayant une hémorragie grave ou une contre-indication à I'utilisation du Minirin ${ }^{\circledR}$, on doit souvent recourir à l'administration de concentré de facteur Willebrand d'origine humaine.

Tous les autres $\mathrm{FH}$ peuvent présenter un déficit d'origine génétique. Parmi ceux-ci, le déficit en facteur XI, relativement fréquent dans certaines populations, est caractérisé par l'absence complète de corrélation entre l'intensité du déficit et la diathèse hémorragique qui, même en cas de déficit sévère, est parfois totalement asymptomatique. Le déficit en facteurs $\mathrm{V}, \mathrm{VII}, \mathrm{X}, \mathrm{II}$ et en fibrinogène se transmet selon le mode autosomal co-dominant. Les formes hétérozygotes avec des taux de facteurs intermédiaires sont en général asymptomatiques ; elles ne sont prises en considération que s'il existe un risque hémorragique important. Les formes sévères peuvent justifier le recours au traitement substitutif (concentrés de facteurs VII, XI, fibrinogène, PPSB, plasma frais congelé).

\section{Revue des protocoles d'hémostase}

(Shurafa et MacIntosh, 1987 ; Sindet-Pedersen, 1986, 1988 ; P iot et coll., 1994 ; B oyar et coll., 1998 ; Galeazzi et coll., 1998 ; Alantar et coll., 1998 ; Stieltjes et coll., 2000 ; De M ello et coll., 2000 ; Makris et coll., 2000 ; Villar et coll., 2000)

Les protocoles proposés sont les suivants :

- Shurafa et MacIntosh (1987) (2 hémophiles ayant un déficit sévère en facteur VIII avec inhibiteurs ; 6 interventions chirurgicales) réalisent les interventions chirurgicales sous anesthésie générale. Ils associent des éponges de gélatine imprégnées de thrombine à du collagène microfibrillaire avec des sutures résorbables au catgut (points croisés) pour rapprocher les berges de la plaie. Une compression pendant au moins une heure est faite à l'aide de compresses stériles. Des coussins thermiques sont appliqués sur les carotid es primitives jusqu'au lendemain. L'hémostase générale est assurée par l'administration de complexe prothrombinique, activé ou non, à raison d'une injection toutes les douze heures, la première injection étant réalisée juste avant l'intervention. Le traitement est prolongé encore médecin€ buccale chirurgie buccale VOL. $11, \mathrm{~N}$ 2005 page 131 
médecine buccale chirurgie buccale

L. $11, \mathrm{~N}^{\circ} 3$

2005

page 132
24 heures après l'arrêt de saignement, puis un relais avec de l'acide epsilon-aminocaprö̈que est institué.

- Sindet-Pedersen $(1986,1988)$ (patients ayant un déficit sévère en $\mathrm{FH}$ dont le nombre n'est pas précisé) réalise une hémostase locale en associant des sutures à une colle biologique et des gouttières hémostatiques. Chez les patients ayant plus de $10 \%$ de taux de $\mathrm{FH}$, la seule administration d'un antifibrinolytique (par voies générale et locale) permet d'obtenir une hémostase efficace sans traitement substitutif.

- P iot et coll. (1994) (20 patients : 3 hémophiles $A$ modérés, 10 hémophiles $A$ mineurs, 1 hémophile $B$ modéré, 1 hémophile $B$ mineur, 1 maladie de Willebrand sévère, 2 maladies de Willebrand mineures, 1 déficit mineur en facteur $V, 1$ déficit mineur en facteur XII ; 26 interventions chirurgicales) associent pour les patients ayant un déficit sévère, thrombine et collagène, sous anesthésie générale ; pour les patients ayant un déficit modéré, collagène-thrombine, oxycellulose-thrombine ou colle gélatine-résorcine-formol ; pour les patients ayant un déficit mineur, thrombine-collagène. La perfusion de CFH est faite pour tous les patients ayant un déficit sévère opérés sous anesthésie générale. Les patients atteints d'hémophilie $A$ mineure ou de maladie de Willebrand bénéficient d'un traitement par la desmopressine. Pour les patients ayant un déficit mineur, modéré ou sévère, l'hémostase a été contrôlée en première intention respectivement pour 7 interventions sur 17,4 sur 6 et 2 sur 3 . La durée moyenne d'hospitalisation est de 2,8 jours, tous déficits confondus.

- Boyar et coll. (1998) (3 patients à déficit sévère avec ACC ; 15 interventions chirurgicales) associent éponges hémostatiques, thrombine, sutures et, pour les avulsions multiples, une gouttière hémostatique. Ils constatent une "réduction importante» des besoins en CFH.

- Hassin et Stieltjes (in Galeazzi et coll., 1998 et Alantar et coll., 1998 ; Stieltjes et coll., 2000) (53 patients; hémophilie : $72 \%$, maladie de Willebrand : $20 \%$, autres déficits : $8 \%, 64$ interventions chirurgicales) associent mèche d'oxycellulose intra-alvéolaire, sutures puis mèche imprégnée de colle cyanoacrylate sur les berges de la plaie ; quand c'est techniquement possible, ils mettent en place des gouttières hémostatiques. Pour les déficits mineurs, il n'y a pas d'administration systématique de CFH. Pour les déficits modérés, l'injection pré-opératoire de CFH est réservée aux interventions invasives; I'administration de desmopressine est systématique pour I'hémophilie $A$ mineure et la maladie de Willebrand. Pour les déficits sévères, l'injection d'une dose pré-opératoire de CFH dans I'heure précédant l'intervention est quasi systématique. L'administration post-opératoire de $\mathrm{CFH}$ est laissée à l'appréciation des praticiens (type d'intervention, profil psychosocial du patient...) avec une attention particulière entre $\mathrm{J} 2$ et $\mathrm{J} 5$. Avec ce protocole, respectivement $100 \%, 66 \%$ et $18 \%$ des interventions ont pu être réalisées, chez les patients ayant un déficit mineur, modéré ou sévère, sans administration de $\mathrm{CFH}$.

- De Mello et coll. (2000) (18 hémophiles : 6 hémophiles $A$ sévères, 5 hémophiles $A$ modérés, 4 hémophiles $A$ mineurs et 3 hémophiles $B$ modérés) associent mèche d'oxycellulose, colle biologique et sutures résorbables ; en post-opératoire, une compression avec de la gaze imprégnée d'acide tranexamique est réalisée toutes les quatre heures pendant 7 jours. Ce protocole comporte également une perfusion pré-opératoire de CFH mais exclut l'utilisation de gouttières hémostatiques. Il a été réactualisé dernièrement (Frachon et coll., 2005) et les auteurs préconisent maintenant : le $1^{\text {er }}$ jour, une compression de 10 min toutes les heures, le $2^{\mathrm{e}}$ jour, une compression de 10 min toutes les deux heures, le $3^{\mathrm{e}}$ jour, une compression de $10 \mathrm{~min}$ toutes les trois heures et le $4^{\mathrm{e}}$ jour, une compression de $10 \mathrm{~min}$ toutes les 4 heures.

- Makris et coll. (2000) (25 patients : 20 hémophiles, 5 maladies de Willebrand; 72 avulsions) associent les gouttières hémostatiques à des sutures, de l'oxycellulose, un silicone occlusif et des bains de bouche avec de l'acide tranexamique. Les perfusions pré-opératoires sont faites avec du facteur VIII ou de la desmopressine. $92 \%$ des patients ont eu une hémostase contrôlée en première intention.

- Villar et coll. (2000) (88 hémophiles : 34 hémophiles sévères, 11 hémophiles modérés, 43 hémo- 
philes mineurs et 7 maladies de Willebrand; 95 interventions pour 208 avulsions) n'effectuent pas de perfusion pré-opératoire mais réalisent une hémostase locale, associant une colle biologique autologue à des sutures et à une compression. $88 \%$ des patients ont eu une hémostase contrôlée ; $9 \%$ des patients un épisode de saignement contrôlé par une reprise de I'hémostase locale (compression et colle) et $3 \%$ des patients un épisode de saignement qui a nécessité une perfusion de CFH.

La revue de la littérature ne permet pas de proposer un protocole standard. La majorité des études ont un NdP de 4 (études retrospectivesséries de cas). Les rares études contrôlées (Walsh et coll., 1971, 1975 ; Forbes et coll., 1972 ; SindetPederesen et Stenberg, 1986 et Stajcic, 1985) sur l'efficacité de l'acide epsilon-aminocaproïque et l'étude cas-témoin sur l'évaluation de l'hémostase locale lors des avulsions dentaires chez les patients hémophiles (Zanon et coll., 2000) ne sont pas randomisées ( $\mathrm{NdP} 2$ ). L'absence d'études randomisées peut se justifier pour des raisons éthiques ; toutefois le caractère contrôlé sur des groupes parallèles (versus groupe sain) permet d'obtenir des preuves satisfaisantes et des résultats cohérents statistiquement exploitables (Zanon et coll., 2000).

La comparaison des résultats des différents protocoles est impossible en raison de l'effet centre et de l'effet temps. Enfin, les critères de jugement sont variables selon les études : pourcentage de patients dont l'hémostase a été contrôlée en première intention (V anczarski et coll., 1990 ; Piot et coll., 1994 ; Waly, 1995 ; De Mello et coll., 2000 ; Makris et coll., 2000 ; Villar et coll., 2000), pourcentage de patients ayant eu un saignement contrôlé par simple reprise de l'hémostase locale sans perfusion de CFH (De Mello et coll., 2000 ; Villar et coll., 2000), quantités de CFH perfusés $\left(\mu \mathrm{g} . \mathrm{kg}^{-1}\right.$ ou U. $\left.\mathrm{kg}^{-1}\right)$ ap rès ré-intervention chirurgicale (Steinberg et Levin, 1984 ; Stajcic, 1985 ; Sindet-Pedersen, 1988 ; Chelappah et Loh. 1990 ; Boyar et coll., 1998 ; Stieltjes et coll., 2000), nombre moyen de jours d'exposition aux CFH (Stieltjes, 2000), nombre moyen de jours d'hospitalisation (P iot et coll., 1994 ; Makris et coll.,
2000 ; Wang et coll., 2000) et, en corollaire, nombre moyen de jours d'arrêt de travail (Makris et coll., 2000). Néanmoins, le groupe de travail a pu à la lumière des données de la littérature répondre aux cinq questions posées initialement.

\section{QUESTIONS POSÉES}

\section{Quelle(s) techniques (s) d'anesthé- sie recommander?}

L'anesthésie locale doit être préférée à l'anesthésie générale (Vinckier et Vermylen, 1985) en raison du risque d'hématome endo-trachéal lié à I'intubation. On réalise une anesthésie para-apicale avec les précautions d'usage (injection vestibulaire, contact doux avec le périoste), éventuellement complétée par une anesthésie intra-ligamentaire pour les secteurs latéro-mandibulaires, avec une aiguille fine $(0,3$ ou $0,4 \mathrm{~mm}$ de diamètre) (Shurafa et MacIntosh, 1987). Une solution de lidocaïne ou d'articaïne de $2 \%$ - $3 \%$ est recommandée (Scully et coll., 2002) ; l'adjonction d'un vasoconstricteur optimise l'hémostase locale et l'effet analgésique (Grellet et Laudenbach, 1985 ; Patton et Webster, 1994 ; Roche, 1996).

L'anesthésie loco-régionale est contre-indiquée si le déficit en FH n'est pas corrigé (Samson et Fiore-Donno, 1987 ; Girard et coll., 1997 ; Evans, 1981 ; Steinberg et Levin, 1984 ; Vinckier et Vermylen, 1985 ; Roche, 1996 ; Scully et coll., 2002 ; B rewer et coll., 2003) ; elle est proscrite en raison du risque d'hématome latéro-pharyngé pouvant provoquer une insuffisance respiratoire aiguë (Gauzeran, 1978 ; Samson et Fiore-Donno, 1987). Une anesthésie loco-régionale, avec aspiration de contrôle, peut être réalisée (avulsion de molaire ou de dents de sagesse incluses, kystecto mie, intervention dans un site inflammatoire) si le trouble de l'hémostase est corrigé (Grellet et Laudenbach, 1985 ; Piot et coll., 1994 ; Roche, 1996 ; Brewer et coll., 2003). Le risque d'hématome profond existe aussi pour les autres techniques d'anesthésie loco-régionale (tubérositaire, infra-orbitaire, technique d'Akinosi) ; S hurafa et MacIntosh (1987) les contre-indiquent sauf en cas de correction complète du déficit. Lors d'une infection locale, une antibiothérapie précède l'antibioprophylaxie [AP]. médecin€ buccale chirurgie buccale VOL. $11, \mathrm{~N}$ 2005 page 133 
médecine buccale chirurgie buccale

L. $11, \mathrm{~N}^{\circ} 3$

2005

page 134
L'anesthésie générale peut être indiquée pour les interventions chirurgicales invasives (dents incluses en position ectopique, avulsions multiples intéressant les différents quadrants, édentation totale bimaxillaire, kystes de grande taille). Les interventions sont alors réalisées en un seul temps, sous réserve d'une correction préalable du trouble de l'hémostase en raison du risque d'hématome endo-trachéal lié à l'intubation (Wang et coll., 2000); la correction est plus ou moins prolongée en fonction de la technique d'intubation [AP]. Les techniques d'intubation bucco-trachéale guidée par fibroscopie et les matériaux utilisés ont nettement diminué le risque d'hématome laryngé ; il est encore diminué par l'utilisation, si l'ouverture buccale le permet, du masque laryngé (Young, 1991 ; Cazalaa et Barrier, 1992 ; Cherfane et coll., 1994). L'anesthésie générale permet aussi de réduire le saignement per-opératoire par l'obtention d'une hypotension contrôlée. L'intubation bucco-trachéale est préférée à l'intubation naso-trachéale qui expose à un risque d'hémorragie nasale pouvant se compliquer d'une inhalation bronchique de sang [AP].

\section{Quelle(s) technique(s) d'avulsion ou de kystectomie utiliser?}

L'avulsion doit être la moins délabrante possible et suivie d'un parage minutieux de la plaie pour éliminer les débris osseux et le tissu gingival inflammatoire (Coulon, 1991). Le saignement peropératoire est plus important lorsqu'on doit réaliser un lambeau que pour l'avulsion de dent(s) sur l'arcade (Ardekian et coll., 2000). S'il est indispensable de pratiquer un lambeau (racines résiduelles ou divergentes, couronnes délabrées, kystectomie...), celui-ci doit être muco-périosté, le périoste richement vascularisé étant soigneusement décollé (Wang et coll., 2000). Selon Shurafa et MacIntosh (1987), l'utilisation de matériel rotatif (fraise à os) doit être limitée pour diminuer le délabrement osseux. La fragmentation de la dent à la fraise Zekrya peut être une bonne alternative à l'alvéolectomie qui est toujours plus délabrante. Pour les avulsions multiples, une régularisation des crêtes (épines irritatives, septum inter-radiculaire) est faite à la rape à os et/ou à la pince gouge fine (Wang et coll., 2000). Une avulsion, avec alvéolectomie vestibulaire et fragmentation de la dent incluse ou d'une dent sur l'arcade, est moins délabrante et moins iatrogène qu'une avulsion prolongée avec manipulation forcée (Wang et coll., 2000).

\section{Quelles techniques hémostatiques locales mettre en œuvre?}

\subsection{Matériaux d'hémostase résorbables}

Ils sont présents dans tous les protocoles et utilisés en association dans la majorité des cas : mèches de cellulose oxydée (Steinberg et coll., 1984 ; Vinckier et Vermulen, 1985 ; Vicens et coll., 1992 ; Galeazzi et coll., 1998 ; Alantar et coll., 1998 ; De Mello et coll., 2000), collagène microcristallin (Evans et coll., 1979 ; Shurafa et Maclntosh, 1987 ; P iot et coll., 1994), éponges de gélatine (Shurafa et MacIntosh,1987; Boyar et coll., 1998) et thrombine (Shurafa et MacIntosh, 1987 ; P iot et coll., 1994 ; B oyar et coll., 1998). Pour les dents pluri-radiculées, l'alvéole de chaque racine doit être comblée individuellement (Boyar et coll., 1998). La thrombine d'origine bovine (thrombase) n'est pas recommandée en raison de son origine et, de plus, elle peut favoriser le développement d'anticorps anti-facteur $\mathrm{V}$ (Streiff et Ness, 2002).

\subsection{Sutures}

Initialement déconseillées (Mulkey, 1976), elles ont montré leur efficacité même pour les avulsions simples car elles réduisent la taille de l'alvéole, assurent une compression, améliorent la tenue du caillot et des matériaux intra-alvéolaires : elles diminuent donc le risque de saignement et améliorent le confort post-opératoire (S tajcic et coll., 1989 ; Patton et Webster, 1994). Après un lambeau muco-périosté, on doit chercher à réaliser une suture étanche en assurant un bon rapprochement des berges (Stajcic et coll., 1989 ; Boyar et coll., 1998) et en pratiquant éventuellement une régularisation osseuse (Boyar et coll., 1989). Certains auteurs (Shurafa et MacIntosh, 1987 ; Coulon, 1991) utilisent du fil résorbable pour les avulsions simples. En chirurgie invasive, 
le fil non résorbable de type soie (3.0) permet de palier les inconvénients de ces sutures à résorption rapide : caractère iatrogène dû au gonflement rapide des fils, durcissement, élimination trop rapide ou au contraire trop lente (Wang et coll., 2000). Différents points de sutures peuvent être utilisés : séparés, en $U$, en $X$; ces derniers sont particulièrement adaptés par leur stabilité et leur force de traction (Shurafa et MacIntosh, 1987). II faut éviter le surjet passé qui cède complètement après la rupture d'un point.

\subsection{Colles et autres moyens d'hémostase locale}

\subsubsection{Colles chimiques}

L'utilisation de la colle gélatine-résorcine-formol ou colle GRF a été abandonnée en raison des difficultés de mise en oeuvre (bain-marie, nécessité d'essorages multiples du formol), de ses contreindications (dents proches des sinus, des fosses nasales, du canal mandibulaire et du trou mentonnier) (Chauvin et coll., 1984 ; Samson et FioreDonno, 1987 ; Bader et coll., 1994) et de ses complications éventuelles (nécrose osseuse induite par le formol); ce risque persiste malgré les modifications apportées à la technique d'utilisation (Commissionat et Croizet, 1982 ; Grellet et Laudenbach, 1989). Elle a cepend ant rendu de grands services pour les praticiens rompus à son utilisation car elle constitue un progrès important pour la réalisation de l'hémostase locale (Vergoz et coll., 1973 ; Henry et coll., 1974 ; Henry, 1976 ; Grellet et Laudenbach, 1989).

Puis ce fut la colle cyanoacrylate. A l'exception de Rozenberg (1984) qui signale un risque de nécrose tissulaire, tous les autres auteurs ont constaté l'absence de risque de nécrose (Pélissier et coll. 1990). Toutefois, Berthier et coll. (2002) rapportent, chez des patients ayant une insuffisance hépatique sévère, deux accidents hémorragiques graves après utilisation de la colle à base de butylcyanoacrylate (Histoacryl $\left.{ }^{\circledR}\right)$ dûs à une nécrose de la muqueuse, rendant toute suture impossible. Stieltjes et coll. (2000), dans une étude portant sur 64 interventions (144 avulsions) chez 53 patients, n'observent aucune complication tissulaire avec la colle embucrylate; ces résultats peuvent s'expliquer par l'interposition entre la mèche imprégnée de colle et l'os alvéolaire d'un matériau intra-alvéolaire. J astrzebski et coll. (1976) qui utilisent la colle à base de cyanoacrylate chez les enfants hémophiles $A$ et $B$ ne rapportent pas cette complication. Enfin de nombreuses applications de cette colle existent en chirurgie cutanée, vasculaire, gastro-intestinale (Qureshi et coll., 1997), cérébrale, orthopédique, esthétique, gynéco-obstétrique et urologique (Cheng et Saing, 1997).

\subsubsection{Colles biologiques}

L'utilisation d'une colle biologique permet de diminuer les épisodes hémorragiques post-avulsionnels et les quantités de CFH perfusés, quel que soit le degré du déficit (Wutka, 1978 ; Martinowitz et Schulman, 1995 ; Wang et coll., 2000). Selon Wang et coll. (2000), pour les avulsions multiples avec un déficit sévère ou modéré, I'utilisation d'une colle biologique permet de pallier à la désunion de la suture secondaire à l'œdème et donc de diminuer la quantité de CFH perfusée.

Un cas de choc anaphylactique fatal a été rapporté après utilisation de Tissucol ${ }^{\circledR}$ chez un patient traité par B-bloquants (Bavoux, 2001); C'est l'aprotinine, antifibrinolytique d'origine bovine, qui serait à l'origine de l'accident allergique. Les accidents par application locale seraient très proches, en gravité, des accidents liés à l'aprotinine bovine par voie intra-veineuse. Les colles biologiques actuellement disponibles sont préparées à partir de plasma humain et contiennent de l'aprotinine d'origine bovine.

Il n'y a pas d'essai contrôlé permettant de comparer l'efficacité des colles biologiques et des colles chimiques.

\subsubsection{Gouttières hémostatiques}

Les gouttières utilisées en France dérivent pour la plupart de celles décrites par Szpirglas et Sultan (in Pélissier et coll., 1990). Elles sont réalisées en résine acrylique autopolymérisable, en pré-opératoire ; en post-opératoire immédiat, elles sont garnies avec une résine auto-polymérisable souple. Bader et coll. (1994) utilisent des gouttières réalisées avec des feuilles de polyuréthane. Toutes ces gouttières présentent plusieurs inconvénients : instabilité en occlusion empêchant toute protec- médecin€ buccale chirurgie buccale VOL. $11, \mathrm{~N}$ 2005 page 135 
médecine buccale chirurgie buccale L. $11, \mathrm{~N}^{\circ} 3$ 2005

page 136 tion efficace du caillot, inconfort, contact souvent traumatisant et augmentation de la dimension verticale responsable d'une hypersalivation qui majore l'effet fibrinolytique de la salive (De Mello et coll., 2000). Afin de palier à ces inconvénients, Hassin et Stieltjes (in Galeazzi et coll., 1998) proposent des gouttières extemporanées qui suppriment la séance préalable d'empreinte. Elles sont mieux adaptées à l'occlusion et à la muqueuse de la crête alvéolaire ; ce qui leur donnent une meilleure stabilité et évite tout traumatisme. Elles sont faites avec un matériau thermoformable. Placées 30 secondes dans de l'eau chaude, les billes de plastique se transforment en une pâte visqueuse permettant une bonne adaptation à l'arcade dentaire et à l'occlusion du patient. Les mouvements réalisés pendant la période de durcissement permettent un ajustage précis. Les excès sont éliminés et, en $5 \mathrm{~min}$, elles deviennent dures et incassables (Galeazzi et coll., 1998). Les gouttières sont déposées à 8 (Solary et Belon, 1999). Wang et coll. (2000) ont utilisé, pour un cas d'édentation totale (31 avulsions), deux gouttières extemporanées avec bourrelet d'occlusion, maintenues à l'aide d'une fronde. Pour l'avulsion des dents de sagesse, on peut réaliser des gouttières extemporanées en silicone lourd avec rebasage éventuel avec du silic one light. Ce matériau qui permet une bonne adaptation, peut être utilisé en milieu humide donc en cas de saignement ; le patient peut même les ôter pour les repas.

L'indication des gouttières hémostatiques est discutée. Pour Piot et coll. (1994), I'hémorragie postavulsion survient plus fréquemment chez des patients dont le taux de $\mathrm{FH}$ est inférieur ou égal à $7 \%$ et qui ne bénéficient pas de gouttières. Les résultats de l'étude de Martinowitz et coll. (1996) (contrôle de l'hémostase chez 88 \% des 106 patients opérés ayant différents déficits en $\mathrm{FH} ; 118$ avulsions) et de De M ello et coll. (2000) (contrôle de l'hémostase chez $85 \%$ des 18 patients ayant différents déficits en $\mathrm{FH}$ ) semblent plaider pour la non utilisation des gouttières quelle que soit la sévérité du déficit. Boyar (1998) réserve les gouttières aux avulsions multiples chez les patients ayant un déficit sévère avec inhibiteur. Les colles peuvent être une alternative quand on est dans l'impossibilité d'utiliser des gouttières (P iot et coll. 1994).

\subsubsection{Antifibrinolytiques}

L'acide tranexamique (Sindet-Pedersen et Stenberg, 1986 ; Baudo et coll., 1988 ; Ramstrom et coll., 1993 ; Waly, 1995 ; Strubbs et Lloyd, 2001) est actuellement l'antifibrinolytique le plus utilisé en chirurgie buccale. Pour mémoire, l'acide epsilon-aminocaproïque (Cooksey, 1966 ; Giordano, 1968 ; Gaum, 1969 ; McIntyre, 1971 ; Short et Ogle, 1974 ; Alagille et coll. 1965 ; Lucas, 1981 ; Evans, 1981, 1982 ; Eastman et coll., 1988 ; Stajcic et coll., 1989) s'est vu retirer son AMM, car son efficacité est 10 fois inférieure à celle de l'acide tranexamique et ses effets secondaires plus importants (Dubber et coll. 1964 ; Forbes et coll., 1972 ; Tavenner, 1972 ; Rizza, 1980 ; Borea et coll., 1993). II n'a pas été trouvé de références bibliographiques concernant l'utilisation de l'aprotinine en chirurgie buccale, hormis le cas de choc anaphylactique rapporté (Bavoux, 2001) qui justifie le choix de l'acide tranexamique. L'administration de l'acide tranexamique est faite par voie générale (3g. $\mathrm{j}^{-1}$ ) (Zanon et coll., 2000) et locale, en rinçage, pour réduire l'activité fibrinolytique du milieu buccal (Bjorlin et Nilsson, 1968 ; Bump et Kolodny, 1973 ; Southan, 1981 ; Moody, 1982 ; Sindet-Pedersen, 1987 ; Sindet-Pedersen et Stenbjerg, 1986, 1988 ; Scully et coll., 2002) ou en imprégnant une mèche ou une compresse avec la solution [AP]. Ils permettent de réduire les doses de CFH utilisés (Walsh et coll., 1971 ; Walsh et coll., 1975 ; Webster et coll., 1973 ; Evans, 1981 ; Statjic, 1985 ; Rodeghiero, 1985 ; Sindet-Pedersen et Stenberg, 1986 ; Stajcic et coll, 1989 ; Vinckier et Vermylen, 1985).

Un protocole utilisant les antifibrinolytiques seuls (par voie générale ou locale), sans perfusion de facteurs, est conseillé chez les patients ayant un taux de FH supérieur à $10 \%$ (Sindet-Pedersen et Stenberg, 1986 ; Sindet-Pedersen, 1988 ; SindetPedersen, 1998). Aucune donnée bibliographique ne permet de généraliser cette pratique aux déficits de FH plus sévères où les antifibrinolytiques sont considérés comme un traitement complémentaire permettant de diminuer les perfusions de CFH et les épisodes hémorragiques post-opératoires (Stajciz, 1985 ; Waly, 1995 ; Martinowitz et coll., 1996 ; Zanon et coll., 2000). 


\subsubsection{Laser $\mathrm{CO2}$}

En expérimentation animale, sur mâchoire de porc, le laser $\mathrm{CO} 2$ a démontré son efficacité dans l'hémostase chirurgicale per-opératoire des tissus mous. Toutefois, cette étude préliminaire n'a pas confirmé son efficacité hémostatique pour le tissu osseux (Ginisty et coll., 1990). Le laser CO2 (15 W) est efficace chez les patients hémophiles A mineurs et modérés dans le traitement des saignements gingivaux (Santos-Dias, 1992 ; Grasser et Ackermann, 1997).

\section{Quelles sont les indications d'un trai- tement par voie générale?}

\subsection{Concentrés de facteurs de l'hémo- stase (CFH)}

L'utilisation des perfusions pré- et post-opératoires de CFH est rapportée par plusieurs auteurs (Evans, 1982 ; Grellet et Laudenbach, 1985 ; Shurafa et MacIntosh, 1987). Les perfusions postopératoires peuvent s'étendre sur une durée de 2 (Shurafa et MacIntosh, 1987) à 10 jours (G rellet et Laudenbach, 1985), à raison de 2 (Shurafa et Maclntosh, 1987) ou 3 injections par jour (Grellet et Laudenbach, 1985). L'évolution s'oriente vers la diminution, voire la suppression, de ces perfusions au profit d'une hémostase locale plus efficace (Sindet-Pedersen, 1988 ; Martinowitz et coll., 1996 ; Stieltjes et coll., 1998 ; De Mello et coll., 2000 ; Villar et coll., 2000 ; Piot et coll., 2003). La plupart des études (Vinckier et Vermylen, 1985 ; De Mello et coll., 2000 ; Stieltjes et coll., 2000 ; Zanon et coll., 2000) recommandent une perfusion unique pré-opératoire ; cette perfusion qui se fait dans l'heure précédant l'intervention, doit permettre d'obtenir un taux de $\mathrm{FH}$ d'environ $30 \%$ (Bartlett et coll., 1985 ; Zanon et coll., 2000 ; Franchini et coll., 2003); les perfusions post-opératoires, non systématiques, sont indiquées en fonction de la sévérité du déficit, du type de chirurgie et de la compliance du patient, et laissées à l'appréciation du chirurgien et de l'hématologue (Vinckier et Vermylen, 1985 ; Sumi et coll., 1985 ; Martinowitz et coll., 1996 ; Stieltjes et coll., 1998, 2000 ; Villar et coll., 2000 ; S tubbs et Lloyd, 2001).
La diminution des perfusions, voire la non-perfusion, de $\mathrm{CFH}$, entraîne une réduction du risque iatrogène (transmission d'éventuels agents méconnus, immunisation contre le facteur perfusé) et permet une prise en charge plus économique (Alantar et coll., 2000).

\subsection{Desmopressine (1-désamino-8-D-argi- nine vasopressine)}

La perfusion, 30 à 60 min avant l'intervention, d'une dose de desmopressine (Minirin ${ }^{\circledR}$; $0,3 \mu \mathrm{g} \cdot \mathrm{kg}^{-1}$ dans 50 à $100 \mathrm{ml}$ de sérum physiologique en injection IV lente, en 15 à $30 \mathrm{~min}$ ), associée à l'administration d'un antifibrinolytique, permet l'avulsion de dents chez les patients bons répondeurs à la desmopressine atteints d'hémophilie $A$ mineure ou de maladie de Willebrand (Abubaker et coll., 1987 ; Leclerc, 1989 ; Solary et Belon, 1999 ; Federici et coll., 2000 ; Stubbs et Lloyd, 2001) sans perfusion de $\mathrm{CFH}$, ni complication hémorragique dans 90 à $100 \%$ des cas (Herrin et Boyd, 1988 ; Mannucci et coll., 1981, 1997 ; Mariani et coll., 1984 ; De la Fuente et coll., 1985; J anczorski et coll., 1990 ; Vicens et coll., 1992 ; Im-Erbsin et Suwannuraks, 1993 ; Piot et coll., 1994 ; Manucci, 1997 ; Stieltjes et coll. 2000 ; Federici et coll., 2000 ; Stubbs et Lloyd, 2001). Elle est réalisée après test au Minirin ${ }^{\circledR}$ positif, avec surveillance du pouls et de la tension artérielle. L'administration de desmopressine peut se faire en spray nasal $\left(\right.$ Cctim $\left.^{\circledR}\right)$. Une surveillance plus rapprochée est nécessaire en cas d'administration répétée. $L a$ desmopressine agit en aug mentant transito irement les taux plasmatiques de facteur VIII et de facteur Willebrand (Stubbs et Lloyd, 2001). Elle est inefficace dans les formes sévères de maladie de Willebrand (type 3 ) et son emploi est théoriquement contre-indiqué $d$ ans la maladie de Willebrand de type 2B. Elle doit être utilisée avec prudence chez les patients présentant une affection cardiovasculaire (hyp ertension artérielle, insuffisance coronarienne). Dans tous les cas, les apports hydriques sont diminués pendant 24 heures en raison de l'effet anti-diurétique. médecin€ buccale chirurgie buccale VOL. $11, \mathrm{~N}$ 2005 page 137 


\section{Comment envisager la surveillance et les traitements post-opératoires?}

\subsection{Hospitalisation}

Les patients ayant un déficit en $\mathrm{FH}$ et nécessitant une intervention de chirurgie buccale, doivent être traités en milieu spécialisé avec un plateau technique adéquat (personnel formé, matériel d'hémostase adapté) (Wilde et coll., 1998 ; Zanon et coll., 2000 ; Scully et coll., 2002; B rewer et coll., 2003). Des complications exceptionnellement gravissimes nécessitant la ligature de l'artère palatine descendante (Wei et coll., 1997) ou celle de la carotide externe (Duarte-Ronces et coll., 1990) ont été rapportées.

La décision de réaliser l'intervention chirurgicale en ambulatoire (Leclerc, 1989) ou en hospitalisation dépend de la gravité du déficit, du type de chirurgie, de la compliance du patient (critères

médecine buccale chirurgie buccale
DCP) et de l'éloignement de son domicile du centre hospitalier. II faut tenir compte du stress et de l'anxiété du patient qui contribuent à l'augmentation des risques de saignements post-opératoires par élévation de la tension artérielle (Mulkey, 1976 ; Wang et coll., 2000 ; Katz et Terezhalmy, 1988). Le nombre moyen de jours d'hospitalisation est de $2,8 \mathrm{j}$ ( $\min : 0$; $\max : 8 \mathrm{j}$.), tous déficits confondus (P iot et coll., 1994).

\subsection{Prescriptions péri-opératoires}

Une antibioprophylaxie per os de 8 jours, débutant en pré-opératoire, est recommandée (Shurafa et MacIntosh, 1987 ; Girard et coll, 1997 ; RPC de la SFAR, 1999 ; Scully et coll., 2002). Elle permet de prévenir une surinfection du site opératoire qui entraînerait une hypervascularisation et une activation de la fibrinolyse (Bump et Kolodny, 1973). Lors du port de gouttières hémostatiques, I'antibioprop hylaxie doit être systématique pour diminuer le risque d'infection favorisée par l'anaérobiose sous-prothétique (Stieltjes et coll., 1998, 2000). Une alimentation semi-liquide, froide ou tiède, et l'absorption de boissons froides pendant au moins 24 heures permettent de limiter les traumatismes du site opératoire et de protéger le caillot (Lewis, 1973 ; Patton et Webster, 1994 ; Roche, 1996 ; De Mello et coll., 2000). L'arrêt du tabac est préconisé pendant la période de cica- trisation pour diminuer le risque d'alvéolite postopératoire (Meechan et coll., 1988).

Une corticothérapie brève, associée à une antibiothérapie, en s'opposant entre autres à la composante vasomotrice de l'inflammation et donc à l'hyperhémie, contribue à diminuer le risque hémorragique (De Mello et coll., 2000). Shurafa et Macintosh (1987) proposent l'application d'un couss in thermique froid sur les carotides primitives [NdP 4]. L'application d'un coussin thermique froid directement sur la région cutanée en regard de la zone opérée, 10 min par heure, dans les 3 premières heures suivant l'intervention est la procédure généralement utilisée [NdP 4]. La prescription d'acide tranexamique est faite $p a r$ voie générale ( 2 à 4 g.j ${ }^{-1} \times 7 \mathrm{j}$ ) (Federici et coll., 2000 ; Zanon et coll., 2000), éventuellement complétée en cas de saignement par son utilisation locale en rinçage (1 ampoule de $10 \mathrm{ml}$ d'acide tranexamique à $5 \%$, pour un rinçage d'une durée de 2 min toutes les 6 heures pendant 7 jours) (Sindet-Pedersen et Stenberg, 1986 ; Ramström et coll., 1993 ; Waly, 1995 ; Strubbs et Lloyd J , 2001 ; Scully et coll., 2002). Les bains de bouche, en particulier ceux ayant un excipient alcoolique (effet hyperhémique), sont proscrits afin de protéger le caillot (Girard et coll., 1997). Les bains de bouche à l'eau glacée peuvent suppléer à cet inconvénient [Grade $\mathbf{C}$ ].

Les antalgiques sont de type paracétamol ou dextropropoxyphène, à l'exclusion des anti-inflammatoires risquant de majorer le saignement (Katz etTerezhalmy, 1988).

\section{CONCLUSION}

Ces recommandations pour les avulsions dentaires et les kystectomies chez les patients présentant un déficit constitutionnel en $\mathrm{FH}$ reposent essentiellement sur des accords professionnels et des données de grade $C$; il n'y a qu'une recommandation de grade $B$ et aucune de grade A. Les 273 publications retrouvées ont un NdP 4 pour leur quasi-totalité ; un article (Zanon et coll., 2000) un NdP 3, un article (Cheng et Saing, 1997) un NdP 2, et aucun article n'a un NdP 1. 
Cette mise au point est une première étape permettant de proposer une conduite à tenir selon un arbre décisionnel. Le protocole d'hémostase doit être adapté à chaque cas et deux éléments déterminent la conduite à tenir : la sécurité et le confort du patient. La sécurité implique de réduire au maximum le risque hémorragique tout en évitant un éventuel effet iatrogène. Un protocole qui permet de limiter, voire de supprimer le recours

\section{RÉFÉRENCES}

1 - Abubaker AO, Bontempo FA, Braun TW. Use of deamino-8-D arginine vasopressin in a patient with moderate Willebrand's disease. J Oral Maxillofac Surg 1987 ; $45: 728-30$.

2 - Alagille D, Lambert A, Laudenbach P, Poulin M. Utilisation de l'acide epsilonaminocap rö̈que dans l'hémostase en stomatologie chez l'hémophile. Rev Stomatol $1965 ; 66: 97$.

3 - Alantar A, Stieltjes N, Sultan Y, Molho P, Princ G, Hassin M. Thermomodelable material called Plastray ${ }^{\circledR}$ used during hemorrhage following dental avulsion (Abstr.). XXIII International Congress of the World Federation of Hemophilia. The Hague, 1998 : 17-22.

4 - Alantar A, Hassin M, Dutheil M, Princ G, Ginisty D, StIELTJ ES N. Coût direct de la prise en charge de patients atteints d'un déficit en faceurs de coagulation et devant subir des avulsions dentaires : étude préliminaire. Méd buc Chir buc $2000 ; 6: 119-20$.

5 - AlEDORT L. Inhibitors in hemophilia patients : current status and management. AmJ Hematol $1994 ; 47$ : 208-17.

6 - Aledort LM, hschmeyer RH, Pettersson ha. Longitudinal study of orthopaedicoutcomes for severe factor-VIII-deficient haemophiliacs. The Orthopaedic Outcome Study Group. J Intern Med 1994 ; 236 : 391-9.

7 - Agence Nationale d'Accréditation et d'Evaluation en Santé. In : Les recommandations pour la pratique clinique. Base méthodologique pour leur réalisation en France. Tipografia Giuntina, Paris, 1999.

8 - Agence Nationale d'Accréditation et d'Evaluation en Santé. In : Analyse de la littérature et gradation des recomandations. Guide méthodologique. Tipografia Giuntina, Paris, 2000.

9 - Bader G, Glez D, De Labarthe C, Gagnot G. Les gouttières thermoformées dans la prévention des hémorragies postavulsionnelles. Actual Odontostomatol 1994 ; 186: 213-9.

10 - Bartlett J A, Sweeney J D, Sadowsky D. Exodontia in combined factor V and factor VIII deficiency. J Oral Maxillofac Surg $1985 ; 43: 537-9$.

11 - Baudo F, De Cataldo F, Landoniao G, Muti G. Management of oral bleeding on haemophilic patients. Lancet $1988 ; 2: 1082$. aux $\mathrm{CFH}$, constitue un atout pour la prévention des risques méconnus liés aux dérivés du sang. Un deuxième atout est d'augmenter l'indice de qualité de vie du patient pendant la phase de cicatrisation. Des études de type coût-efficacité chez les patients présentant un déficit en $\mathrm{FH}$ et nécessitant une intervention de chirurgie buccale devraient permettre de valider les techniques d'hémostase proposées.

12 - Bavoux F. Pharmacovigilance des médicaments dérivés du sang. Procès verbal du Comité Local du Médicament (CLM), Groupe Hosiptalier Cochin-Saint Vincent de Paul $2001: 7$.

13 - Berthier AM, Guilly Gomarc'h A, Messner M, Pommereuil M, Bader G, De Mello G. Use of recombinant factor VIla to treat persistent bleeding followwing dental avulsions in two cirrhotic patients. Vox Sang $2002 ; 82: 199-201$.

14 - B J ORLIN G, NILSSON IM. Fibrynolytic activity in alveoli after tooth avulsion. Odontol Rev $1968 ; 19$ : 197-204.

15 - BJ ORLIN G, NILSSON IM. Tooth avulsion in hemophiliacs after ad ministration of a single dose of factor VIII or factor IX concentrate supplemented with AMGA. Oral Surg Oral Med Oral Pathol $1973 ; 36: 482-9$.

16 - B linder D, Martinowitz U, Varon D, Steinberger A, TAICHER S. Intraoral persistent bleeding in hemophilia A patients-the role of clots removal. XXIII International Congress of the World Federation of Hemophilia. Haemophilia $1998 ; 4$ : 304.

17 - Borea G, Montebugnoli, Capuzzi P, Magelli C. Tranexamic acid as a mouthwash in anticoagulant-treated patients undergoing oral surgery Oral Surg Oral Med Oral Pathol $1993 ; 75: 29-31$.

18 - Boyar R, SCHWETZ N, J OHnSton J. Dental surgical management of hemophilia patients with inhibitors. Haemophilia $1998 ; 4$ : 305.

19 - Brewer AK, Roebuck EM, Donache M, Hazard A, Gordon K, Fung D, CLARKSON J. The dental management of adult patients with haemophilia and other congenital bleeding disorders. Haemophilia $2003 ; 9$ : 673-7.

20 - Bump RL, Kolodny SC. Fibrinolysis: a possible factor in the control of postoperative hemorrhage in the patient with hemophilia Oral Surg $1973 ; 36: 195-9$.

21 - Cadenat H, Fabie M, Bernes A, Authier J P. Hemostasis disorders and tooth avulsions, our technic for local hemostasis. Rev Stomatol Chir Maxillofac 1973; 74 : 686-9.

22 - Cazalaa J B, Barrier G. Aspects pratiques du masque laryngé. Cahiers Anesthésiol $1992 ; 5$ : 361-5. médecine buccale chirurgie buccale VOL. $11, \mathrm{~N}$ 2005 page 139 
23 - Chellappah NK, Loh HS. Laser therapy for a haemophiliac. Case report. Aust DentJ $1990 ; 35: 121$.

24 - CHENG W, QAING H. a prospective randomized study of wound approximation with tissue glue in circumcision in children. J Paediatr Child Health 1997 ; 33 : 515-6.

25 - Cherfane A, Yazbeck P, Antakly MC. Le masque laryngé : un nouvel outil pour l'anesthésiste. Cahiers Anesthésiol $1994 ; 1: 35-40$.

26 - ChIono O. Dental anesthesia for hemophilic patient. Anesth Prog $1968 ; 15: 295-8$.

27 - Commissionat Y, Croizet J . Les avulsions dentaires chez les malades soumis aux anticoagulants. InfDent 1982 ; $64: 3799-808$.

28 - CoOksey MW, Perry CB, Raper AB.Epsilon-aminocaproic acid therapy for dental avulsion in haemophiliacs BrMed J $1966 ; 2: 1633-4$.

29 - Coulon J P. Pathologie cutanéo-muqueuse. (pp776854). In : Traité de pathologie buccale et maxillo-faciale, E Piette, H Reychler. éd : De Boeck-Wesmael, Bruxelles, 1991.

30 - De la Fuente B, Kasper CK, Rickles FR, Hoyer LW. Response of patients with mild and modelate haemo- médecine buccale chirurgie buccale

L. $11, \mathrm{~N}^{\circ} 3$

2005

page 140 philia $A$ and Willebrand's disease to treatment with desmopressin. Ann Intern Med $1985 ; 103: 6-14$.

31 - De Mello G, Pommereuil M, Petition M, Berthier AM. Local haemostasis without use of splint in the management of dental avulsions in haemophilia. Haemophilia $2000 ; 6: 408-12$.

32 - Duarte-Ronces H, Hernandez-Cazares L, GutierrezROMERO M. Fracture of the maxillary tuberosity in the hemophiliac patient. Pract Odontol $1990 ; 11: 15-25$.

33 - Dubber AH, McNicol GP, Douglas PS. Some properties of the antifib rinolytically active isomer of amino methylcyclohexane carboxilic acid. Lancet $1964 ; 2$ : 1317.

34 - Eastman J R, Nowakowski AR, TRIPLett DA. DDAVP: review of indications for its use in the treatment of factor VIII deficiency and report of a case. Oral Surg Oral Med Oral Pathol $1983 ; 56: 246-51$.

35 - Emis G, Kavaklî K, Polat A, Ertugrul F, Aydînok Y, Cetingül E, Nisli G, Kantar M, Cetingu N, Ozto D. Dental care and current therapy in turkish hemophiliacs. Haemophilia $1998 ; 4$ : 305.

36 - Evans BE, IRVING SP, Aledort LM. Use of microcristallin collagen for hemostatis after oral surgery in a haemophiliac. J Oral Surg $1979 ; 37$ : 126-8.

37 - Evans BE. The use of epsilon aminocaproic acid as an adjunct to replacement therapy in hemophiliacs undergoing tooth avulsion. Part I. N Y J Dent 1981 ; 51 : 8390.

38 - Evans BE.The use of epsilon aminocaproic acid as an adjunct to replacement therapy in hemophiliacs undergoing tooth avulsion. Part II. N Y J Dent $1981 ; 51$ : 11727.

39 - Evans BE. Hemophilia in the child and adult. Masson, New York, 1982.
40 - Evans BE. Local hemostatic agents (and techniques). Scand J Haematol $1984 ; 33: 417-22$.

41 - Federici AB, Sacco R, Satbile F, Carpenedo M, Zingaro E, MannUCCI PM. Optimising local therapy during oral surgery in patients with von Willebrand disease: effective results from a retrospective analysis of 63 cases. Haemophilia $2000 ; 6: 71$.

42 - Forbes CD, Barr RD, Reid G, Thomson C, Prentice CR, MCNicol GP, Douglas AS. Tranexamic acid in control of haemorrhage after dental avulsion in haemophilia and Christmas disease. $\mathrm{Br}$ Med J $1972 ; 2: 311-3$.

43 - Franchini M, Rossetti G, Tagliaferri A, Pattacini C, Pozzoli D, Lippi G, Manzato F, Bertuzzo D, Gandini G. Effic acy and safety of fac tor VII/von Willebrand's factor concentrate (Haemate-P) in preventing bleeding during surgery or invasive procedures in patients with von Willebrand disease. Haematologica $2003 ; 88$ : 127983.

44 - Galeazzi J M, Alantar A, Hassin M, Stieltjest N. Utilisation d'un matériau thermoformable, le Plastray ${ }^{\circledR}$, lors d'hémorragies post-avulsionnelles. Méd Buc Chir Buc 1997 ; 3 : 83-8.

45 - GAUM LI. Use of aminocaproïc acid in oral surgery for haemophiliacs. Report of case. J Can Dent Assoc $1969 ; 35: 599-602$

46 - Gauzeran D. L'hémorragie : sa prévention en chirurgie buccale ( $p 43$ ). Polycopiés d'odonto-stomatologie. J ulien Prélat, Paris, 1978

47 - Ginisty D, Gineste P, Bonneau M, Drouet L, Lavergne A. Etude expérimentale de l'effet hémostatique du Laser CO2 chez le porc atteint d'une maladie de Willebrand. Laser Med Surg $1990 ; 6: 81-7$.

48 - GIORdano ND, WatKINS RS, RadivoYeVITCH M, GoodstITT E. Dental avulsion in hemophilic patients on amino-caproïc acid prophylaxis. Further experience with two additional cases. Oral Surg Oral Med Oral Pathol $1968 ; 26: 160-6$.

49 - Girard P, Quevauvilliers J , J eandot J , Perlemuter L. Dictionnaire médical du chirurgien-dentiste (pp 335336). Masson, Paris, 1997.

50 - Goudemand J. Hémophilies.Enyc Médicochir 13021B10, 18 p, 1997.

51 - Grasser H, Ackerman K. Possibilities for the use of lasers in dental surgery. Dtsch Zahnarzh Z 1997 ; 32 : 512-15.

52 - Grellet M, Laudenbach P. Thérapeutique stomatologique et maxillo-faciale (pp156-60) Masson, Paris, 1984.

53 - GuYATt GH, SACKett DL, Sinclair J C, HaYward R, Cook DJ , Cook RJ . User' guides to the medical literature. IX. A method for Grading Health Care Recommendations. J Am Med Ass 1995 ; 274 : 1800-4.

54-Heim MU, Lutze G, Aumann V, Schumacher J, FREIGANG B. Postoperative haemorrage in a girl with congenital factor $X I$ deficiency-successful treatment with desmopressin (DDAVP, Minirin ${ }^{\circledR}$ ). Klin Padiatr $2002 ; 214: 128-31$. 
55 - Henry F, Kelemen ZS, Vergoz D, Laufer J, Grignon J L. La colle GRF en stomatologie. Avulsions dentaires chez les malades présentant des troubles de l'hémostase. Rev Stomatol Chir Maxillofac $1974 ; 75: 609-12$.

56 - HenRY F. L'hémostase des avulsions dentaires par la colle GRF. Rev S tomatol Chir Maxillofac 1976 ; 2 : 307-9.

57 - HERRIN RA, Boyd J F. Desmopressin acetate prophylaxis in a patient with hemophilia A: report of case. J Am Dent Assoc $1988 ; 117: 593-4$.

58 - IFI J C, BENOIT M. Gouttières hémostatiques souples utilisées pour les avulsions dentaires chez les hémophiles. Rev Prothèse Maxillofac $1977 ; 6: 218-22$.

59 - IM-ERBSIN T, SuWANNURAKS M. Local hemostatic technic using a celluloid splint in bleeding disorders. Southeast Asian J Trop Med Public Health 1993 ; 24 : 167-8.

60 - J AnCZARski M, Ruszkowska I, Cetnarowicz H, LoPACSUK S. Intravenous adminis tration of deamino-Darginine-vasopressin (DDAVP) to patients with hemophilia and Willebrand's disease. Acta Haematol Pol $1990 ; 21: 16-21$.

61 - Jastrzebski S, Dyszy-Laube B, Kurnatowski W, Brzozolowki T, SzUmilewicz-Serocka B, Kaminska D. Tooth avulsion in children with hemophilia $A$ and $B$, using dressings of cyanoacrylic surgical glue. Czas Stomatol $1976 ; 29: 787-92$.

62 - J ONES J , HUNTER D. Qualitative research: consensus methods for medical and health services research. $\mathrm{Br}$ Med J $1995 ; 311: 376-80$.

63 - KatZ J O, Terezhalmy GY. Dental management of the patient with hemophilia. Oral Surg Oral Med Oral Pathol $1988 ; 66: 139-44$

64 - LECLERC D. Etude de la maladie de Willebrand et nouvelles données thérapeutiques en odonto-stomatologie. Inf Dent $1989 ; 21$ : 1781-91.

65 - LewIS B. Dental care for the haemophiliac. J Am Dent Assoc $1973 ; 87: 1411-5$.

66 - LoREnZETTI MA. Dental avulsion in the hemophiliacs, Méd Hyg $1968 ; 26: 1107$.

67 - LUCAS ON, ALBERT TW. Epsilon aminocaproic acid in hemophiliacs undergoing dental avulsions: a concise review. Oral Surg Oral Med Oral Pathol 1981; $51: 115$.

68 - LURIE A, SILVERMAN NH, J ACKSON FR. An approach to dental avulsion in hemophilia and related bleeding disorders in children. S Afr Med J $1972 ; 46$ : 1743-6.

69 - LURIE R, SMITH I, LURIE A. Dental avulsion in haemophilic patients. J Dent Assoc S Afr $1977 ; 32$ : 517-20.

70 - Makris SP, FoKa Z, Pithara E, Makris PE.Non-bleeding tooth avulsions in haemorragic patients. Haemophilia $1998 ; 4: 303$.

71 - Makris SP, Foka Z, Pithara E, Makris PE.Comparaison of all the applied methods for the local haemostasis in tooth avulsion in haemorrhagic patients. Haemophilia, $2000 ; 6: 409$.

72 - M ANNUCCI PM. Desmopressin (DDAVP) in the treatment of blooding disorders: The first 20 years. Blood 1997 ; $90: 2515-21$.
73 - Mannucci PM, Canciani MT, Rota L, Donovan BS. Response of factor VIII/von Willebrand factor to DDAVP in healthy subjects, and patients with haemophilia $A$ and Willebrand's disease. BrJ Haematol 1981 ; 47 : 283-93.

74 - Mannucci PM, Ruggeri ZM, Pareti FI, Capitanio A. Deamino-8-d-arginine vasopressin: a new pharmacological approach to the management of haemophilia and Willebrand's disease. Lancet $1997 ; 1:$ 689-72.

75 - Mariana G, Ciavarella N, Mazzucconi MG, Antoncecchi S, Solinas S, Ranieri P, Pettini P, AgRESTINI F, MANDELLI F. Evaluation of the effectiveness of DDAVP in surgery and in bleeding episodes in haemophilia and Willebrand's disease. A study on 43 patients. Clin Lab Haematol 1984 ; 6 : 229-38.

76 - MARTINOWITZ U, SCHULMAN S. Fibrin sealant in surgery of patients with hemorrhagic diathesis. Thromb Haemost $1995 ; 74:$ 486-92.

77 - Martinowitz U, Schulman S, Horoszowski H, Heim M. Role of sealants in surgical procedures on patients with hemostatics disorders. Clin Orthop $1996 ; 328: 65-75$.

78 - M CINTYRe H. L'utilisation de l'acide epsilon-aminocaprö̈que chez les hémophiles. Rev S tomatol Chir Maxillofac $1971 ; 72: 330-32$.

79-Meechan JG, Mac Gregor DM, Rogers SM, Hobson RS, Bates J PC, DEnNisson M. The effect of smoking on immediate post-avulsion socket filling with blood and on the incidence of painful sockets. $\mathrm{Br}$ J Oral Maxillofac Surg $1988 ; 26$ : 402-9.

80 - Moody CR. P lasminogen in human saliva. Int J Oral Surg $1982 ; 11: 110-3$.

81 - MULKEY TF. Outpatient treatment of hemophiliacs for dental avulsions. J Oral Surg $1976 ; 34: 428-34$.

82 - Patton LL, Webster WP. Bleeding and clotting disorders, (pp 544-62). In: Burket's Oral Medicine, MA Lynch, VJ Brightman, MS Greenberg. ed: J B Lippincoll Company, Philadelphia, 1991.

83 - Pelissier A, Arnault N, Pelissier-Gele B. Hémorragies d'origine dentaire : les techniques loc ales d'hémostase. Actual Odontostomatol $1990 ; 170: 307-22$.

84 - Peterson J. Tranexamic acid to reduce hemorrhage in hemophiliacs (letter). J Oral Maxillofac Surg 1988; 46 : 176

85 - Piot B, Fiks-Sigaud M, Feri J , Gordeef A, Mercier J . Les avulsions dentaires chez les hémophiles et les porteurs de la maladie de Willebrand. Rev Stomatol Chir Maxillofac $1994 ; 95$ : 263-7.

86 - Piot B, Sigaud-Fiks M, Huet P, Fressinaud E, Trossaert M, Mercier J . Avulsions dentaires chez les patients porteurs d'un trouble de l'hémostase. Proposition d'un protocole basé sur le type d'anesthésie nécessaire. Rev Stomatol Chir Maxillofac 2003 ; $104: 73-6$.

87 - Qureshi A, Drew PJ, Duthie GS, Roberts AC, MONSON J R. N-butylcyanoacrylate adhesive for skin closure of abdominal wounds : preliminary results. Ann $R$ Coll Surg Engl $1997 ; 79: 414-15$. médecin€ buccale chirurgie buccale VOL. $11, \mathrm{~N}$ 2005 page 141 
88 - Ramstrom G, Sindet-Pedersen S, Hall G, Blomback M, AlANDER U. Prevention of postsurgical bleeding in oral surgery using tranexamic acid without dose modification of oral antic oagulants. J Oral Maxillofac Surg $1993 ; 51: 1211-6$.

89 - Recommandations pour la pratique de l'antibioprophylaxie en chirurgie. Société Française d'AnesthésieRéanimation. Actualisation des recommandations de la conférence de concensus de décembre 1992.

90 - Rizza CR. Inhibitors of fibrinolysis in the treatment of hemophilia. J Clin Pathol $1980 ; 33: 50-4$.

91 - RoCHE Y. Chirurgie dentaire et patients à risque (pp281301). Flammarion, Paris, 1996.

92 - Rodeghiero F. Treatment of hemophilia. Ric Clin Lab $1985 ; 15: 289-303$.

93 - Rodeghiero f, Castaman G, Dini E. Epidemiological investigation of the prevalence of von Willebrand disease. Blood $1987 ; 69: 454-59$.

94 - Rozenberg C. La colle G.R.F. Actual Odontostomatol 1984 ; 146 : 291-6.

95 - Sacco R, Stablile F, Carpenedo M, Cafro am, MostaRda G, Federici AB. Local therapy during oral surgery in patients with von Willebrand disease. A

médecine buccale chirurgie buccale retrospective analys is of 46 cases. Haemophilia 1998 ; $4: 305$.

96 - Sadler J E, Mannucci PM, Berntorp E, Bochkov N, Boulyjenkov V, Ginsburg D, Meyer D, Peake I, Rodeghiero F, S rivastava A. Impact, diagnosis and treatment of von Willebrand disease. Thrombosis Haemostasis $2000 ; 84: 160-4$.

97-SAMSON J, FIORE-Donno G. Chirurgie buccale et troubles de I'hémostase. Quest Odontostomatol $1987 ; 12: 373-80$.

98 - Santogostino E, Mannucci PM, Bianchi Bonomi A. Guidelines on replacement therapy for haemophilia and inherited coagulation disorders in Italy. Haemophilia $2000 ; 6: 1-10$.

99 - SANTOS-DiAS A. CO2 laser surgery in hemophilia treatment. J Clin Laser Med Surg $1992 ; 10$ : 297-301.

100 - S Cully C, Watt-S mith P, Dios PD, Giangrande PLF. Complications in HIV-infected and non-HIV-infected haemophiliacs and other patients after oral surgery. Int J Oral Maxillofac Surg $2002 ; 31: 634-40$.

101 - SHORT SG, OGLE RG. Avulsions in hemophilia. Use of EACE. Minn Med $1974 ; 57: 77-80$.

102 - ShuRAfa M, Macintosh RB. Management of dental avulsions in two hemophilia A patients with factor VIII inhibitor. J Oral Maxillofac Surg 1987 ; 45 : 698-701.

103 - Sindet-Pedersen S, Ingerslev J, Ramstrom G, BLOMBACK M. Management of oral bleeding in haemophilic patients (Letter). Lancet $1988 ; 3: 566$.

104 - Sindet-Pedersen S. Prevention and treatment of oral bleedings. Haemophilia $1998 ; 4: 302$.

105 - Sindet-Pedersen S, StenBj eRg S. Effect of local antifibrinolytic treatment with tranexamic acid in hemophiliacs undergoing oral surgery. J Oral Maxillofac Surg $1986 ; 44: 703-7$.

106 - SINDET-PederSEN S. Distribution of tranexamic acid and plasma and saliva after oral administration and mouth rincing : a pharmacokinetic study. J Clin Pharmacol $1987 ; 27: 1005-8$.

107 - Sindet-Pedersen S, Stenbjerg S, Ingerslev J . Control of gingival hemorrhage in hemophilic patients by inhibition of fibrinolysis with tranexamic acid. J Periodont Res $1988 ; 23: 72-4$

108 - SOLARY E, BELON J P. Thérapeutique pour le pharmacien (pp78-85). In Hématologie. Masson, Paris, 1999.

109 - SonIS AL, Musselman RJ . Oral bleeding in classic hemophilia. Oral Surg $1982 ; 53: 363-6$.

110 - SOUTHAN J C. The fibrinolytic activity of human oral epithelium and epidermis. BrJ Dermatol $1981 ; 105$ : 679-83.

111 - STAJ CIC Z. The combined local/systemic use of antifibrinolytics in hemophiliacs undergoing dental avulsion. Int J Oral Surg $1985 ; 14: 339-45$.

112 - Staj cic Z, Baklaj a R, Elezovic I, Rolovic Z. Primary wound closure in haemophiliacs undergoing dental avulsions. Int J Oral Maxillofac Surg $1989 ; 18$ : 14-6.

113 - Steinberg SE, LeVIN J , BeLL WR. Evidence that less replacement therapy is required for dental avulsions in hemophiliacs. am j hematol $1984 ; 16: 1-13$.

114 - Stieltjes N, Alantar A, Molho P, Sultan Y, Princ G, HASSIN M. Econometrics and management of patients with bleeding disorders undergoing teeth avulsions : a preliminary study (Abstr.). Haemophilia 1998 ; 4 : 304.

115 - Stieltjes N, Alantar A, Molho P, Hassin M. Clotting factor concentrate requirement for teeth avulsion in patients with congenital bleeding disorders : 7-years experience of a strategy based on enhanced local haemostasis study. Haemophilia $2000 ; 6: 410$.

116 - STREIFF MB, NeSS PM. Acquired FV inhibitors: a needless iatrogenic complication of bovine thrombine exposure. Transfusion $2002 ; 42: 18-26$.

117 - StUBbs M, LLOYd J . A protocol for the dental management of Willebrand's disease, haemophilia A and haemophilia B. Aust Dent J $2001 ; 46$ : 37-40.

118 - SUMI Y, SHIKIMORI M, KanedA T, KITA IMA T. Multiple avulsions in a patient with factor VII deficiency. J Oral Maxillofac Surg $1985 ; 43: 382-4$.

119 - TAVENNER RWH. Use of tranexamic acid in control of haemorrage after avulsion of teeth in haemophilia and Christmas disease. Br Med J 1972 ; 2 : 314-5.

120 - Vergoz D, Henry F, Kelemen ZS, Laufer J , Grignon J L. Controle du saignement pathologique après avulsion dentaire par application de colle gélatine-résorcine formol (méthode Kelemen). Nouv Presse Méd 1973; $26: 1459-60$.

121 - Vicens G, Mathiot J L, Mettoudi J D, Malllet S, KONG KY B, G INISTY D. Intérêts et limites de l'utilisation de la desmopressine (Minirin) en stomatologie. Rev Stomatol Chir Maxillofac $1992 ; 93$ : 127-9.

122 - Villar CC, Regazzo J T, Castro FPMS, Renno HW, Corte AL, Grigolon CC, Borges ACM, Vermiglio AaV, 
Aniquino-BizZachi] M, ArRuda VR, Correa MEP. The use of autologous fib rin glue in oral chirurgical procedures without replacement therapy. A prospective study. Haemophilia $2000 ; 6: 411$.

123 - VinCKIER F, Vermylen J . Dental avulsions in hemophilia: reflections on 10 years' experience. Oral Surg Oral Med Oral Pathol $1985 ; 59: 6-9$.

124 - Walsh PN, Rizza CR, Matthews J M, Eipe J, Kernoff PB, Coles MD, Bloom AL, Kaufman BM, Beck P, HANAN CH, BIGGS R. Epsilon-aminocaproic acid therapy for dental avulsions in haemophilia and Christmas disease: a double blind controlled trial. $\mathrm{Br}$ J Haematol $1971 ; 20: 463$.

125 - Walsh PN, Rizza CR, Evans BE, Aledort LM. The therapeutic role of epsilon aminocaproic acid (EACA) for dental avulsions in hemophiliacs. Ann NY Acad Sci $1975 ; 240: 240-67$.

126 - WALY NG. Local antifibrinolytic treatment with tranexamic acid in hemophilic children undergoing dental avulsions. Egypt Dent J 1995; 41 : 961-8.

127 - Wang C, Kelche S, Gangloff P. Maxillary and mandibulary total toothlessness in a severe haemophiliac: techniques and results. Haemophilia $2000 ; 6: 411$.

128 - WARRIER I, LUSHER J M. DDAVP: A useful alternative to blood components inmoderate hemophilia $A$ and Willebrand disease. J Pediatr 1983 ; 102 : 228-33.

129 - Webster WP, McMilLan CW, LuCAS ON, ROBERTS HR. Dental management of the bleeder patient. A comparative review of replacement therapy (p.33). In: Haemophilia. F Ala, KWE Denson. ed: Expercta Medica, Amsterdam, 1973.
130 - Wei DC, Wong RW, Robertson EP. Congenital factor VII deficiency presenting as delayed bleeding following dental avulsion. A review of the role of factor VII in coagulation. Pathology $1997 ; 29$ : 234-7.

131 - WILDE J T, COOK RJ . Von Willebrand disease and its management in oral and maxillofacial surgery. $\mathrm{Br} J$ Oral Maxillofac Surg $1998 ; 36: 112-8$.

132 - WUTKA P. Experience with the fibrin adhesive system in the care of avulsions wounds in hemophiliacs. Osterr Z Stomatol 1978 ; 75: 450-2.

133 - Young TM. The lanyngeal mask in dental anaesthesia. EurJ Anaesthesiol 1991 ; Suppl 4: 53-9.

134 - Zanon E, Martinelli F, Bacci C, ZerbinatiP, Girolami A. Proposal of a standard approach to dental avulsion in haemophilia patients. A case control-study with good results. Haemophilia 2000 ; 6 : 533-6.

NDLR : après la recherche bibliographique qui couvrait la période 1966-2003, d'autres articles sont parus, dont celuiCi :

Frachon $X$, Pommereuil M, Berthier AM, Lejeune S, HourdinEude S, Quero J, Meziere X, De Mello G, Garnier J. Management options for dental extraction in hemophiliacs: a study of 55 extractions (2000 - 2002). Oral Surg Oral Med Oral Pathol Oral Radiol Endod $2005 ; 99: 270-5$ médecin€ buccale chirurgie buccale VOL. 11, N 2005 page 143 